\title{
Unique functions for Notch4 in murine embryonic lymphangiogenesis
}

\author{
Ajit Muley ${ }^{1} \cdot$ Minji Kim Uh $^{1,2}$. Glicella Salazar-De Simone ${ }^{1}$ Bhairavi Swaminathan ${ }^{3} \cdot$ Jennifer M. James $^{4}$. \\ Aino Murtomaki ${ }^{1,5,6}$. Seock Won Youn ${ }^{3}$. Joseph D. McCarron ${ }^{1}$. Chris Kitajewski ${ }^{1}$. Maria Gnarra Buethe ${ }^{1}$. \\ Gloria Riitano $^{1,7} \cdot$ Yoh-suke Mukouyama $^{4} \cdot$ Jan Kitajewski ${ }^{3} \cdot$ Carrie J. Shawber ${ }^{1,8}(\mathbb{0}$
}

Received: 17 February 2021 / Accepted: 8 October 2021 / Published online: 19 October 2021

(c) The Author(s) 2021

\begin{abstract}
In mice, embryonic dermal lymphatic development is well understood and used to study gene functions in lymphangiogenesis. Notch signaling is an evolutionarily conserved pathway that modulates cell fate decisions, which has been shown to both inhibit and promote dermal lymphangiogenesis. Here, we demonstrate distinct roles for Notch4 signaling versus canonical Notch signaling in embryonic dermal lymphangiogenesis. Actively growing embryonic dermal lymphatics expressed NOTCH1, NOTCH4, and DLL4 which correlated with Notch activity. In lymphatic endothelial cells (LECs), DLL4 activation of Notch induced a subset of Notch effectors and lymphatic genes, which were distinctly regulated by Notch1 and Notch4 activation. Treatment of LECs with VEGF-A or VEGF-C upregulated Dll4 transcripts and differentially and temporally regulated the expression of Notchl and Hes/Hey genes. Mice nullizygous for Notch4 had an increase in the closure of the lymphangiogenic fronts which correlated with reduced vessel caliber in the maturing lymphatic plexus at E14.5 and reduced branching at E16.5. Activation of Notch4 suppressed LEC migration in a wounding assay significantly more than Notch1, suggesting a dominant role for Notch4 in regulating LEC migration. Unlike Notch4 nulls, inhibition of canonical Notch signaling by expressing a dominant negative form of MAML1 (DNMAML) in Prox1+ LECs led to increased lymphatic density consistent with an increase in LEC proliferation, described for the loss of LEC Notch1. Moreover, loss of Notch4 did not affect LEC canonical Notch signaling. Thus, we propose that Notch4 signaling and canonical Notch signaling have distinct functions in the coordination of embryonic dermal lymphangiogenesis.
\end{abstract}

Keywords Lymphangiogenesis · Notch $\cdot$ VEGF-C · Dermis

\section{Introduction}

Lymphangiogenesis is the process by which new lymphatic vessels sprout off pre-existing vessels. Sprouting of new lymphatic vessels requires coordinated lymphatic endothelial

Ajit Muley and Minji Kim Uh have contributed equally to this work.

Carrie J. Shawber

cjs2002@cumc.columbia.edu

1 Department of Obstetrics and Gynecology, Columbia University Medical Center, New York, NY 10032, USA

2 Department of Pharmacology, Columbia University Medical Center, New York, NY 10032, USA

3 Department of Physiology and Biophysics, University of Illinois Chicago, Chicago, IL 60612, USA

4 Laboratory of Stem Cell and Neuro-Vascular Biology, Cell and Developmental Biology Center, National Heart, Lung, and Blood Institute, National Institutes of Health, Bethesda, MD 20892, USA
5 Wihuri Research Institute, Biomedicum Helsinki, Haartmaninkatu, 8, 00290 Helsinki, Finland

6 Translational Cancer Medicine Program, Faculty of Medicine, Helsinki Institute of Life Science, University of Helsinki, FI-00014 Helsinki, Finland

7 Departments of Molecular Medicine and Experimental Medicine, Sapienza University, 00185 Rome, Italy

8 Department of Surgery, Columbia University Medical Center, New York, NY 10032, USA 
cell (LEC) proliferation, directional migration, and cell-cell adhesion to form a properly patterned and functional network. In murine dorsal skin, lymphangiogenesis begins at embryonic day 12.5 (E12.5) at the side of the trunk and follows dermal blood vessel development to meet at the midline around E15.5 (Fig. S1a) [1]. Dermal lymphangiogenesis in mouse embryos is well characterized allowing for analysis of lymphatic endothelial signaling pathways, such as Notch.

The Notch family of signaling proteins consists of four cell surface receptors (NOTCH1-4) that are bound and activated by membrane-bound ligands of the Delta-like (Dll1, 4) and Jagged (Jag1, 2) families expressed on neighboring cells. Upon ligand activation, the extracellular domain of $\mathrm{NOTCH}$ is released, which induces conformational changes that expose two proteolytic cleavage sites (TACE and $\gamma$-secretase/presenilin) that in turn releases the intracellular cytoplasmic domain (NICD) from the cell surface [2]. In the canonical Notch signaling pathway, NICD transits to the nucleus, binds the transcriptional repressor RBPjk, where it recruits an activation complex including Mastermindlike (MAML) and HDACs, and activates RBPjk-dependent transcription of Notch effectors, such as those in the HES/ Hey families. Notch also signals via a less well-understood non-canonical RBPjк-independent pathway that has been suggested to not require nuclear localization of NICD [2].

During development of the blood vascular system, Notch signaling is essential for arterial endothelial specification, vascular smooth muscle cell differentiation and viability, and sprouting angiogenesis [3-5]. Studies of murine retinal angiogenesis have shown that VEGF-A, via activation of VEGFR2, upregulates DLL4 expression in the filopodiaextending tip cell located at the vascular front [4, 6-8]. Dll4 signals to the neighboring Notch-expressing stalk cell, where Notch activation downregulates VEGFR2 and VEGFR3 expression and inhibits the tip cell phenotype. During retinal angiogenesis, inhibition of DLL4 or NOTCH1 leads to a hypersprouting phenotype characterized by an increase in tip cells at the expense of the stalk cells, increased VEGFR2 and VEGFR 3 expression, and decreased vascular outgrowth $[6,7,9]$. Although it has been shown that VEGF-C induces DLL4 in cultured LECs $[10,11]$, the mechanisms by which Notch regulates dermal lymphangiogenesis remain to be elucidated.

We previously demonstrated that NOTCH1 and NOTCH4 are expressed in the postnatal maturing dermal lymphatics [12]. Studies of postnatal lymphangiogenesis have shown that pharmacological inhibition or genetic manipulation of Dll4/Notch1 signaling can result in both increased and decreased lymphangiogenesis [11, 13]. Neutralizing antibodies against NOTCH1 or DLL4 suppressed lymphangiogenesis in the postnatal mouse ear, tail dermis, and a wounding model [13]. In contrast, an inhibitory soluble DLL4 extracellular domain fused to FC (Dl14FC) stimulated lymphangiogenesis in the postnatal mouse ear [11]. In embryonic dermal lymphangiogenesis, Notch1 deletion in LECs did not affect lymphatic branching, but increased lymphatic vessel caliber which was proposed to be secondary to an increase in LEC proliferation and decreased LEC apoptosis [14]. More recently, it was shown that loss of one copy of Dll4 was associated with reduced embryonic dermal lymphangiogenesis in mice [15], a phenotype opposite to that seen in retinal angiogenesis [7, 8]. Additional studies are needed to clarify the differences in the lymphangiogenic phenotypes observed upon disruption of lymphatic endothelial Notch signaling.

Here, we examined the roles for Notch4 and canonical Notch signaling in embryonic dermal lymphangiogenesis. We demonstrated that NOTCH1, NOTCH4, and DLL4 are expressed, and Notch signaling active in embryonic dermal lymphatic endothelium. VEGF-A and VEGF-C signaling differentially regulated Notch pathway gene expression and activity in cultured LECs. Mice nullizygous for Notch4 displayed an embryonic dermal lymphangiogenic phenotype characterized by increased LEC migration and reduced branching. In contrast, inhibition of canonical Notch signaling increased lymphatic vascular density consistent with an increase in LEC proliferation. Together, these data demonstrate that dermal lymphangiogenesis is dynamically regulated by Notch and requires both NOTCH1 and NOTCH4 functions, as well as canonical and non-canonical Notch signaling.

\section{Materials and methods}

\section{Cell culture/constructs}

HeLa cells were maintained in 10\% FBS DMEM. Human umbilical vein endothelial cells (HUVEC) were isolated as previously described and maintained in EGM2 (Lonza) $[16,17]$. Neonatal human dermal lymphatic endothelial cells (HdLECs) were either purchased (Promocell) or isolated as previously described [18] and maintained on fibronectincoated plates in EGM2-MV2 (Lonza; complete medium) supplemented with $10 \mathrm{ng} / \mathrm{mL}$ VEGF-C (R\&D). To activate Notch signaling, HdLECs were lentivirally infected [19] using pCCL.pkg.wpre vector encoding N1IC, N4/Int-3, or GFP. N1IC encodes the constitutively active cytoplasmic domain of NOTCH1. N4/Int-3 encodes an activated Notch4 allele generated by MMTV insertion [20]. Transcripts and protein expression was confirmed by quantitative (q)RT-PCR and Western analyses of samples collected post-infection. 


\section{HdLEC assays}

VEGF treatment of HdLECs: confluent HdLEC monolayers were starved overnight in 1\% FBS in EBM2 (Lonza) or in human endothelial SFM (Fisher Scientific) followed by either 1 or $5 \mathrm{~h}$ in EBM2/SFM containing 100ng/mL VEGF-A (R\&D), 100ng/mL VEGF-C (R\&D), or 500ng/ $\mathrm{mL}$ VEGF-C $\mathrm{C}^{\mathrm{Cl} 156 \mathrm{~S}}$ (R\&D) prior to RNA isolation. Assays were performed at least 3 times. For detection of AKT and ERK activity, HdLECs were serum starved overnight in SFM containing $1 \%$ FBS and $0.1 \%$ BSA, followed by $5 \mathrm{~h}$ in SFM alone. Cells were then switched to SFM containing $0.1 \%$ BSA and either $100 \mathrm{ng} / \mathrm{mL}$ VEGF-A or $100 \mathrm{ng} / \mathrm{mL}$ VEGF-C for 20 min prior to fixation with cold 4\% PFA. Assays were performed in duplicate for two different lentiviral transductions.

Migration Assay: HdLECs were seeded in triplicate on a fibronectin-coated (Thermofisher) 12-well plate in complete medium. The following day (0-hour time-point), a scratch through the confluent monolayer was made across each well using a $200 \mu \mathrm{l}$ pipet tip, and medium was changed to EBM2 containing $100 \mathrm{ng} / \mathrm{ml}$ VEGF-C. For migration assays with mitomycin $\mathrm{C}$, confluent monolayers were pretreated with $10 \mu \mathrm{g} / \mathrm{mL}$ mitomycin-C (Sigma) for $45 \mathrm{~min}$ prior to scratching. Cells were maintained in EBM2 containing 100ng/ml VEGF-C and $0.1 \mu \mathrm{g} / \mathrm{mL}$ mitomycin-C while migration was assessed. Growth into the scratch was documented at $0,4,8$, 12 , and $25 \mathrm{~h}$ with a Zeiss Axiovert $40 \mathrm{CSL}$ inverted microscope or at $0,4,8$, and $24 \mathrm{~h}$ using an Olympus IX83 microscope. Cell migration rate was determined using imageJ software [21] and calculated as the percentage of cell-free area at different time-points relative to the initial wound area. Assays were performed at least 2-3 times for two independent lentivirally generated HdLEC populations.

\section{Co-culture Notch reporter assay}

HdLECs (90\% confluent) were lipofected (Lipofectamine 2000; Invitrogen) with the Notch reporter plasmid pGL3.11CSL [12] containing 11 repeats of Notch/CSL (RBPjк) cis-elements, and phRL-SV40 renilla (Promega) to normalize lipofection efficiency. HeLa cells were lipofected with pCR3 plasmids encoding either DLL4-FLAG or JAG1FLAG with empty vector serving as a control. $24 \mathrm{~h}$ after lipofection, HeLa and HdLECs were co-cultured together at a 1:1 ratio on fibronectin-coated plates in EGM2. $24 \mathrm{~h}$ after co-culture, a luciferase reporter assay was performed using the Dual-Luciferase Reporter Assay System (Promega) and a TD20/20 luminometer (Turner Designs). Luciferase values were normalized to Renilla values. Each condition was performed in triplicate, 4 times.
DLL4-ligand activation assay and mRNA sequencing

Tethered Ligand Assay: The recombinant extracellular domain of the Notch ligand hDLL4FC (Sino Biologicals Inc.) or IgG-FC (Sino Biologicals Inc.) were coated onto a 24-well plate (Corning) on a fibronectin matrix (Sigma). Following an overnight incubation at $4{ }^{\circ} \mathrm{C}$, primary ECs (at $80 \%$ confluency) were trypsinized and seeded onto the coated plates and incubated at $37{ }^{\circ} \mathrm{C}$ with $5 \% \mathrm{CO}_{2}$ for $6 \mathrm{~h}$. Experiment was performed in triplicate.

RNA was isolated using the RNEasy Mini Kit (Qiagen), quantity and integrity measured using a Bio-analyzer (Agilent TapeStation 4200, UIC Genome Research core) prior to RNA sequencing. TLA HdLEC samples were sequenced at a $\sim 30$ million paired-end (PE) read depth with 150-base fragments by Novogene (https://en.novogene.com/). Raw reads from in vitro screens were mapped to the Human database (ENSEMBL/GRCh38) using STAR (version 2.5.0a) and processed with Samtools (version 1.4.1). The counts obtained by FeatureCounts (version 1.5.2) were analyzed by DESeq2 (version 1.18.1) to identify differentially expressed genes. The RNAseq datasets generated in the current study are available in the NCBI Gene Expression Omnibus repository at https://www.ncbi.nlm.nih.gov/geo (Accession Number GSE183631).

\section{Gene expression analyses}

RNA was isolated using the RNEasy Mini Kit (Qiagen) and reverse transcribed using the VersoTM cDNA Synthesis Kit (Thermo Fisher) or First Strand Synthesis Kit (Invitrogen). qRT-PCR was performed in triplicate for each gene (Table S1), using ABsoluteTM Blue QPCR SYBR Green Master Mix (Thermo Fisher) or Sybr Green Master Mix (Applied Biosystems) and 7300 Real-Time PCR System (Applied Biosystems) or CFX96 PCR Cycler (Biorad). Gene-specific qRT-PCR standards were used to determine transcript levels and normalized to $\beta$-actin expression [12]. PCRs were set up in triplicate and performed at least 3 times.

For the validation of mRNA sequencing data, qPCR was done using SYBR Green master mix (Applied Biosystems) and primers specific to genes of interest (Table S2). The mean cycle threshold $(\mathrm{Ct})$ values from the triplicate run for each sample were analyzed using $\beta$-actin as the reference gene. $\Delta \Delta \mathrm{Ct}$ method [22] was used to calculate the relative expression using the following steps: (1) Normalization to reference gene: $\Delta \mathrm{Ct}_{\mathrm{GOI}}=\mathrm{Ct}_{\mathrm{GOI}}-\mathrm{Ct}_{\mathrm{BA}}$. (2) Relative expression between conditions: $\Delta \Delta \mathrm{Ct}_{\mathrm{GOI}}=\Delta \mathrm{Ct}_{\mathrm{EXP}}-\Delta \mathrm{Ct}_{\mathrm{CNT}}$. The analysis was done using Microsoft Excel and Prism. 


\section{Western blotting}

NOTCH4 expression in Notch4 null mice was determined by Western blot. Fresh E14.5 hindlimbs were lysed in RIPA buffer (Invitrogen) containing protease and phosphatase inhibitors (Thermofisher) on ice and protein concentration determined by BCA protein assay kit (Pierce). Equal amounts of protein were separated on an $8 \%$ SDS-PAGE gel and transferred to a nitrocellulose membrane. The membranes were blocked with $3 \%$ nonfat milk and $3 \%$ bovine serum albumin (Jackson ImmunoResearch) in Tris-Buffered Saline Tween-20 and probed with antibodies against the cytoplasmic domain of NOTCH4 [23] and $\beta$-ACTIN (Abclonal). Horse radish peroxidase-conjugated secondary antibodies (Life Technologies) were used, detected with SuperSignal West Pico Chemiluminescent Substrate (Pierce) and images captured with Biorad Chemdoc MP.

\section{Mouse studies}

Notch4 nullizygous ( $\left.\mathrm{N}^{-/-}\right)$[24], ProxlCreER ${ }^{T 2}$ [25], and $D N M A M L^{f l f l}$ [26], CBF:H2B-Venus (NVR reporter purchased from Jax Labs) [27] and Proxl-tdTomato lymphatic reporter (ProxTom) [28] mice were used for these studies. Studies were performed in mice with a mixed background, as well as a pure C57BL6j background. For studies using ProxlCreER ${ }^{T 2}$, tamoxifen in corn oil was administered via oral gavage $(10 \mathrm{mg} / 40 \mathrm{~g})$ at E12.5. 3 or more independent litters were assessed for each analysis. Number of embryos analyzed is presented in the figure legends.

\section{Immunohistochemistry \& Imaging}

E14.5 and E16.5 dorsal skin was dissected, fixed for $2 \mathrm{~h}$ in $4 \%$ PFA, and then immunostaining initiated. Alternatively, embryos were incubated overnight in 4\% PFA and then stored in $1 \times$ PBS at $4{ }^{\circ} \mathrm{C}$. E14.5 tissues were incubated for $2 \mathrm{~h}$ at room temperature in blocking buffer $(10 \%$ donkey serum, $0.3 \%$ Triton X-100, $1 \times$ PBS), incubated in primary antibody (Table S3) diluted in blocking buffer overnight at $4{ }^{\circ} \mathrm{C}$, and then incubated with the appropriate Alexa Fluor secondary antibody (Invitrogen) diluted in blocking buffer overnight at $4{ }^{\circ} \mathrm{C}$. E16.5 dermal tissues were washed in $1 \times$ PBS containing $0.2 \%$ Triton X-100 and $20 \%$ DMSO for $4 \mathrm{~h}$ at room temperature and immunohistochemistry performed as described in Cha et al. 2016 [29]. For immunostaining of sections, 5-micron sections were stained as previously described [30]. Tissue was mounted using Vectashield with and without DAPI (Vector Laboratories). Images were captured using a Nikon SMZ-U Zoom 1:10 microscope and Nikon 4500 digital camera, Nikon ECLIPSE E800 microscope and NIS Elements software, Nikon DXM 1200 digital camera, and Image ProPlus v.4.01 software, a Zeiss Axioskop2 Plus and Zeiss AxioCam MRc camera with Zeiss Zen software, or an Olympus IX83 Inverted System Microscope and Olympus cellSens software. Confocal microscopy was performed with a Zeiss LSM 510 META Confocal Microscope and the LSM software.

For cell immunochemistry, cells were fixed in $4 \%$ PFA on ice for $15 \mathrm{~min}$, permeabilized and blocked with $0.1 \%$ Triton X-100, 2\% BSA, 3\% donkey serum in $1 \times$ PBS for $1 \mathrm{~h}$ at room temperature. Cells were then incubated overnight with primary antibody at $4{ }^{\circ} \mathrm{C}$ followed by an incubation with Alexa Fluor-conjugated anti-donkey secondary antibodies (Invitrogen) for $1 \mathrm{~h}$ at room temperature. Slides were washed in $1 \times$ PBS and mounted with Vectashield with DAPI mounting media. Images were captured with an Olympus IX83 Inverted System Microscope and Olympus CellSens software.

Images were analyzed with ImageJ or Adobe Photoshop. Tiled 10x images were used to quantify lymphatic and blood vascular density, distance between migration fronts, fronts per unit length, and branch-point per unit length. 20x images were used to determine lymphatic vessel caliber, distance to first branch-point, number of Prox $1+$ cells per field, and sprout morphology. Vascular density was determined as positive signal area normalized by total area. Distance between migrating fronts was determined as the mean distance between the 2 lymphatic fronts measured at multiple points ( $\geq 3$ ) [1]. For analysis of migration of Notch4 mutants, Notch $4^{+/-}$embryos were used to normalize between litters, as they were present in all litters analyzed and the distances between migration fronts were not statistically different than WT embryos. Sprouting fronts, defined as the sprouts that reside at the leading edge of the migrating front per unit length was determined as the number of sprouting fronts at the leading edge of the migration front normalized to the vertical length (posterior-anterior; Fig. S1a). Length of the sprout was determined as length from tip of sprout at lymphangiogenic front to the first branch-point. Lymphatic vessel caliber was determined by measuring the width of lymphatic vessels in the maturing lymphatic plexus and adjacent to the first branch-point from the migrating front. Sprout morphology at the lymphangiogenic front was determined by counting total number of blunt-ended sprouts (rounded, lacking multiple filopodia) and spiky-ended sprouts (elongated with multiple filopodia) normalized to the total number of sprouts assessed. Branch-points per unit length in maturing lymphatic plexus was determined as the number of branch-points per field normalized to the total length of lymphatic vessels per field. To measure Prox1+ LEC number, Prox 1+/LYVE1+ LECs were scored and mean number per field determined. To determine the significance between control and one experimental group, a two-tailed student's $t$ test was used. For analyses of more than two groups, oneway analysis of variance (ANOVA) was used to determine 
significance by unpaired $t$ test. For analyses of multiple conditions and cell populations, two-way ANOVA was used and Dunnett's multiple comparison test performed to determine significance between groups. A $p<0.05$ was considered significant.

\section{Lymphangiography}

Lymphangiographies were performed as described on E17.5 embryos [15]. Briefly, $2 \mu \mathrm{L}$ of $0.4 \%$ Trypan blue solution (Sigma) was injected into the dermis in periorbital region with a $36 \mathrm{G}$ beveled needle attached to a Nano 1 syringe (WPI). The embryos were imaged using AMSCOPE stereomicroscope (AMSCOPE) with camera attachment $1 \mathrm{~min}$ after injection.

\section{Results}

\section{Embryonic dermal lymphatics expressed NOTCH1, NOTCH4, and the Notch ligand, DLL4}

Cultured human LECs, HdLECs, express NOTCH1-4 and the Notch ligands, DLL4 and JAGGED1 (JAG1) [18], while NOTCH1 and NOTCH4 are expressed in the postnatal day 4 (P4) murine dermal lymphatic vessels [12]. Notch signaling has been shown to be active in the E15.5 dermal lymphatics [14], but it is not known which Notch proteins and ligands are expressed in dermal LECs at this time. To study the role of Notch signaling in embryonic dermal lymphangiogenesis, we determined the expression of NOTCH1 and NOTCH4, and the angiogenic Notch ligands, DLL4 and JAG1, as well as Notch activity in E14.5 dorsal skin. This time-point is characterized by the presence of two LYVE1+ lymphatic fronts migrating toward the midline which precedes a maturing lymphatic plexus (Fig. S1a). At this time-point, two $\mathrm{CD} 31+$ angiogenic fronts have fused at the midline to form a connected blood capillary network.

At E14.5, DLL4 was expressed in both in the developing lymphatics and blood vessels of the dermis (Fig. 1a). Unlike the retina, where DLL4 expression is restricted to 1-2 tip cells at the angiogenic front [6-8], high DLL4 expression was observed in multiple LECs within the sprouts at the lymphangiogenic front. DLL4 was also expressed in the arterial vessels and blood capillary network, with strongest expression observed in the large arteries, consistent with its expression in the vasculature of the intestinal villi [10]. Unlike DLL4, JAG1 was not expressed in dermal lymphatics at E14.5 (Fig. 1a). JAG1 expression was limited to the blood vasculature, where the highest expression was observed in the larger caliber arteries in a pattern consistent with vascular smooth muscle cell and endothelial cells. Staining for NOTCH4 and NOTCH1 demonstrated that they were both expressed through-out the endothelium of the sprouts at the lymphatic front which overlapped with DLL4 (Fig. 1b, c). Analysis of E14.5 dermal cross-section confirmed that the LYVE1+ dermal lymphatic endothelium expressed both NOTCH1 and NOTCH4 (Fig. S1b, c). Outside of the lymphatics, NOTCH1 expression was observed in the epidermis and blood endothelium, while NOTCH4 was expressed in the epidermis and a subset of LYVE1+ macrophages (Fig. 1b, c, Fig. S1b, c) .

To determine where Notch is actively signaling during lymphangiogenesis, the dermal lymphatics in E14.5 embryos carrying alleles for the Prox 1-tdTomato (ProxTom) LEC reporter [28] and the Notch Venous Reporter (NVR) [27] were assessed. Notch activity was observed throughout the lymphatic vascular plexus at both the lymphangiogenic front, defined as the LECs that make up the sprout from tip to the first branch-point, and the mature plexus where the vessels have begun to remodel (Fig. 2a). At the lymphangiogenic front, Notch activity was often observed in several LECs located at the tip cell positions in spiky-ended sprouts with filopodia (Fig. 2b), consistent with the broad expression of NOTCH1, NOTCH4, and DLL4 at the front (Fig. 1). Notch activity was also observed in blunt-ended sprouts. In the mature lymphatic plexus, Notch activity was observed at branch-points (Fig. 2a, c). Taken together, the expression data suggest that DLL4 signaling via NOTCH1 and/or NOTCH4 has a role in regulating dermal lymphangiogenic growth and maturation.

\section{Profiling of DLL4/Notch signaling in human dermal lymphatic endothelial cells}

Expression studies suggested that DLL4 is the major ligand for Notch signaling in the lymphatic endothelium during dermal lymphangiogenesis. To determine if DLL4 or JAG1 could activate Notch in LECs, co-culture assays were performed in which endogenous Notch activation was determined using a Notch-response CSL luciferase reporter [12]. HeLa cells were engineered to express DLL4, JAG1, or both (Fig. S2a) and then seeded at a 1:1 ratio with HdLECs containing a CSL-luciferase reporter. DLL4-expressing HeLa cells upregulated Notch signaling nearly five-fold over co-cultures using parental HeLa cells, while JAG1 only modestly increased Notch signaling in HdLECs (Fig. S2b). Co-culture with HeLa cells co-expressing DLL4 and JAG1 induced Notch signaling similar to the co-cultures with DLL4 alone, suggesting that JAG1 did not interfere with DLL4 signaling. Together with the expression studies, these data suggest that DLL4 functions as a ligand for LEC NOTCH.

To further assess DLL4/Notch signaling, HdLECs were seeded on DLL4FC-coated or FC-coated (control) plates. After $6 \mathrm{~h}$, RNA was collected and mRNA sequencing 
A

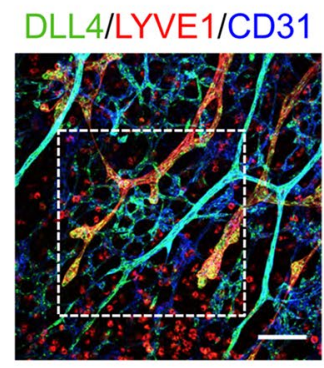

JAG1/LYVE1/CD31

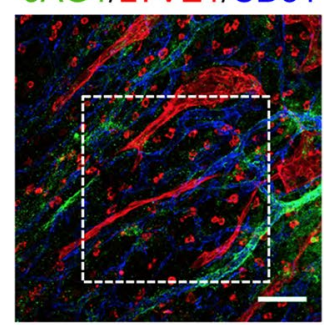

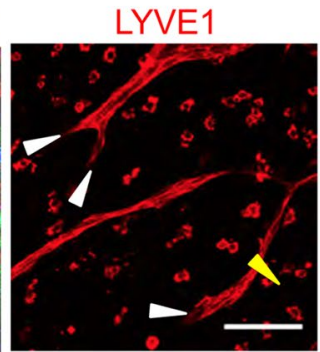

LYVE1

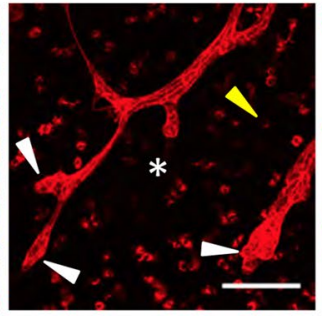

LYVE1
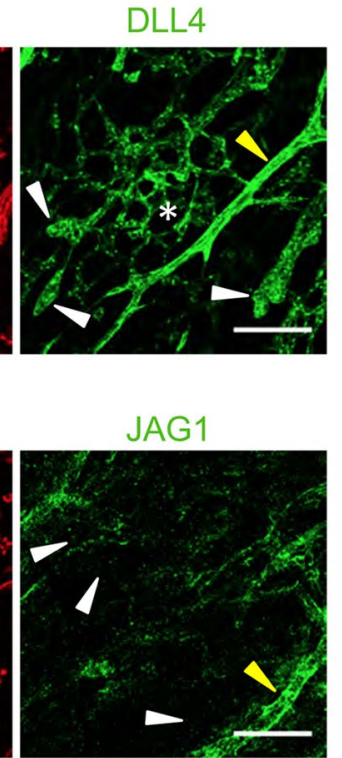

B

ProxTom

C DLL4/NOTCH1
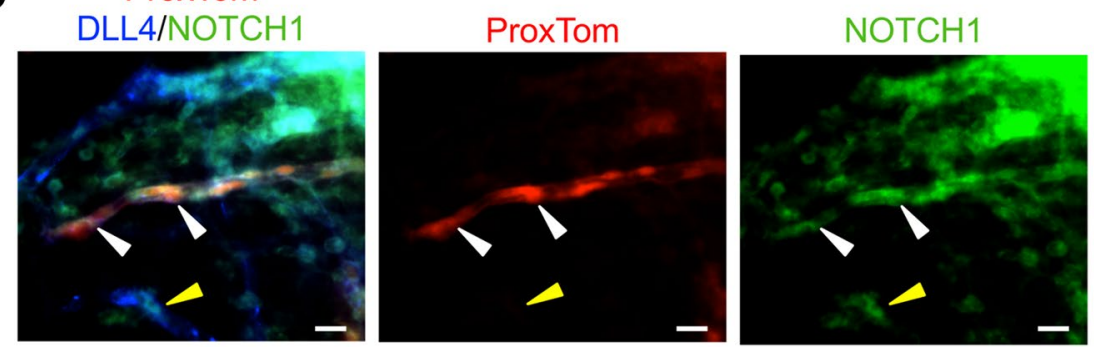
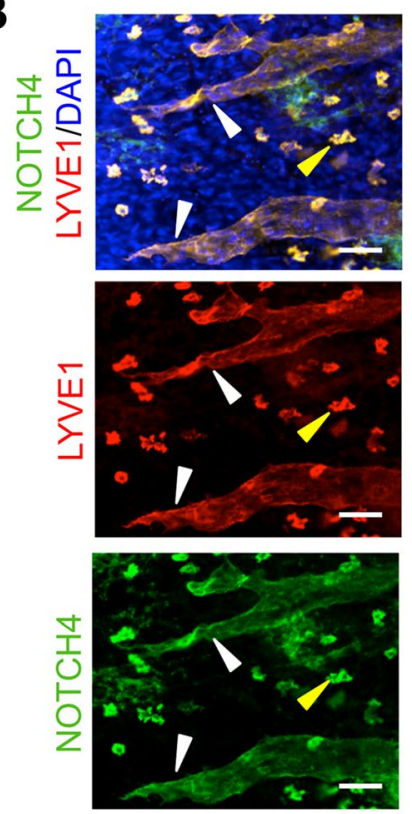

Fig. 1 Embryonic dermal lymphatics expressed NOTCH1, NOTCH4, and DLL4. a E14.5 wild-type skin wholemounts stained for LYVE1, CD31 and DLL4 or JAG1. Higher magnification of boxed areas presented to the right. White arrowheads mark sprouts at the lymphangiogenic front. White asterisk marks the blood vascular plexus. Yellow arrowhead marks an artery. Scale bars, $100 \mu \mathrm{m}$. b E14.5 wild-type skin wholemount stained for LYVE1 and NOTCH4. White arrowheads mark lymphatics at the front. Yellow arrowhead marks a NOTCH4+ macrophage. Scale bars, $50 \mu \mathrm{m}$. c E14.5 ProxTom skin wholemount stained for DLL4 and NOTCH1. White arrowheads mark lymphatic sprout at the front. Yellow arrowhead marks NOTCH1+/DLL4+ blood vessel. Scale bars, $20 \mu \mathrm{m}$

signaling induces genes responsible for pattern specification, neurogenesis, and chemotaxis (Fig. 3d).

To assess if DLL4-induced genes were downstream of Notch 1 or Notch 4 activation, HdLECs were generated to express activated forms of NOTCH1 (N1IC) or NOTCH4 (N4/Int-3). Quantitative RT-PCR was performed for Notch effectors of the Hes and Hey gene families, as well as lymphangiogenic genes. Of the Notch effectors assessed, N1IC and N4/Int-3 both induced the expression of Hes1, Hes4, $H e y 2$, and $H e y L$, with the strongest induction observed for Hes4 and HeyL (Fig. 3e). In contrast, only N1IC induced the expression of Hes5. Notch1 and Notch4 activation significantly induced the expression of the majority of lymphangiogenic genes assessed, except for $C x c r 4, B m p 2$, and Tgfrb2 (Fig. 3f). Similarly, both suppressed the expression of Proxl, Podoplanin, and Lyvel (Fig. S3b, S4b). Cxcr4 was significantly induced by N1IC, while N4/Int-3 suppressed 
Fig. 2 Notch activation observed throughout the embryonic dermal lymphatic vascular plexus. E14.5 ProxTom;NVR skin wholemounts stained for LYVE1. a Low magnification image demonstrating Notch activity throughout the developing lymphatic plexus. Blue arrowheads mark sprouts at the lymphangiogenic front with Notch activity. White arrowheads mark regions of high Notch signaling in the maturing plexus. Scale bars, $500 \mu \mathrm{m}$. b High magnification of spiky-ended lymphatic sprout. White arrowheads mark tip cells with Notch activity. Yellow arrowheads mark stalk cells with Notch activity. c High magnification of the maturing plexus. White arrowheads mark LECs with Notch activity. b, c Scale bars, $100 \mu \mathrm{m}$
A
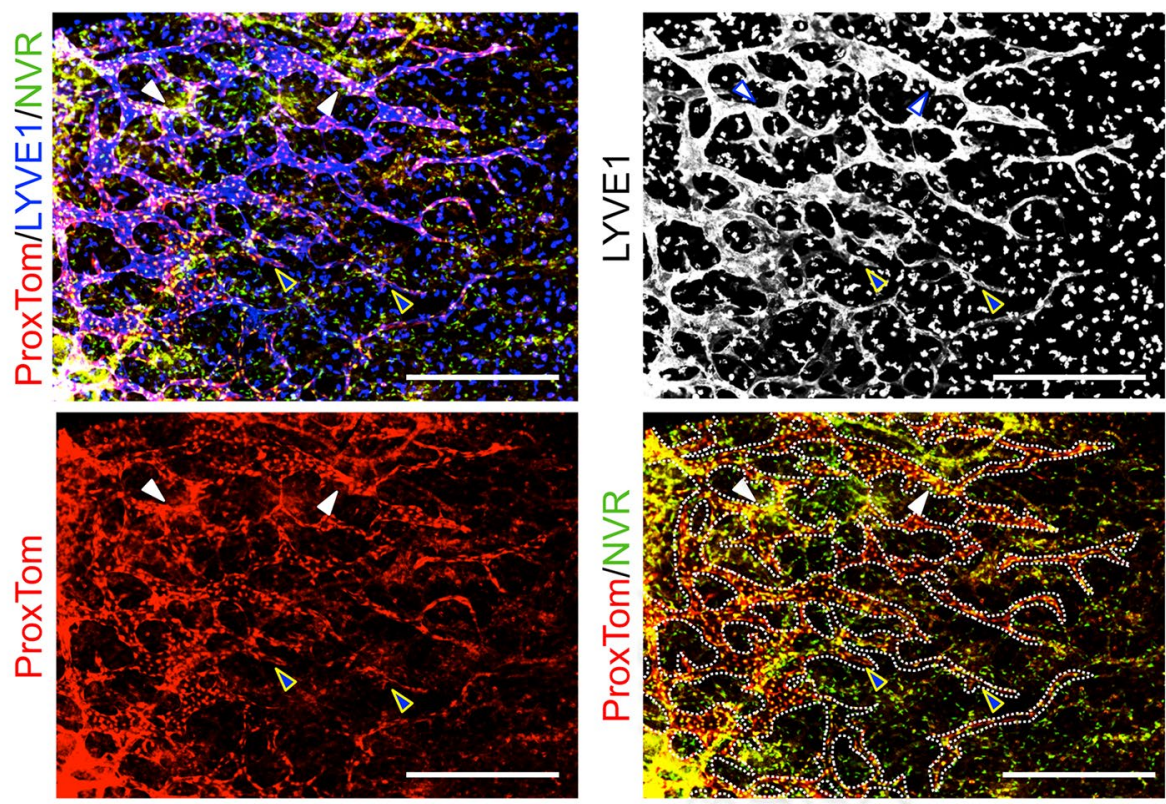

B

Lymphangiogenic front
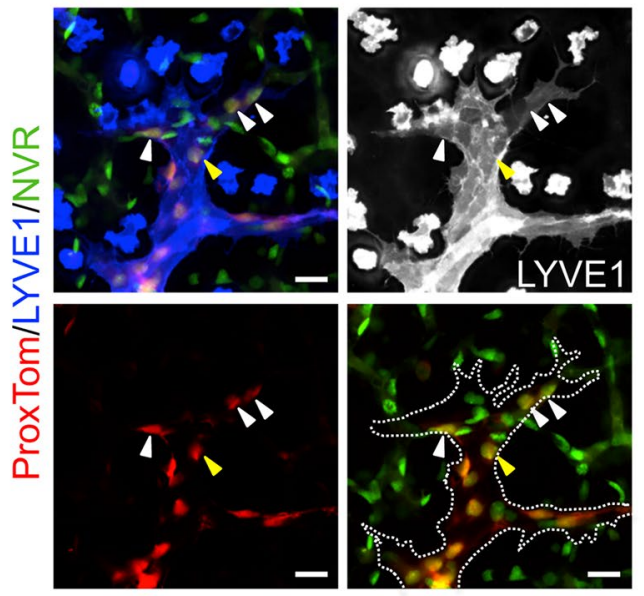

C

Maturing plexus
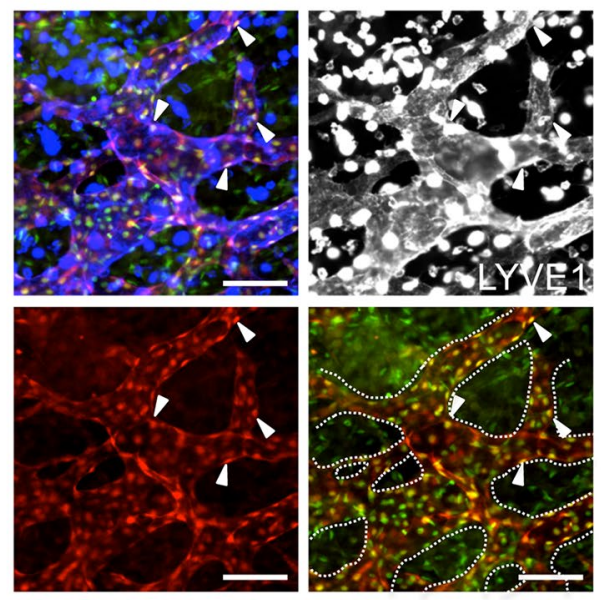

its expression. Neither Tgfrb2 and Bmp2 were induced. While $A c k r 3$ and $C c l 2$ were significantly induced by N1IC relative to the GFP controls, N4/Int-3 was a much stronger inducer of both these genes $(p<0.0003, p<0.0001 \mathrm{~N} 4 /$ Int-3 vs. N1IC, respectively). Together these data support overlapping and distinct downstream signaling for Notch1 and Notch4 in LECs.

\section{VEGF-C induced DII4 expression and Notch activation in HdLECs}

During sprouting angiogenesis, VEGF-A/VEGFR-2 signaling upregulates DLL4 in blood endothelial tip cells to activate Notch signaling in the adjacent stalk cell [6-8]. As we observed DLL4 expression and Notch activity in LECs of the sprouts located at the lymphangiogenic front, we determined the effect of VEGF-A and VEGF-C on Notch genes, ligands, and effectors in HdLECs. Serum-starved HdLECs were treated with either VEGF-A, VEGF-C or VEGF-C ${ }^{\mathrm{C} 156 \mathrm{~S}}$. In HdLECs, VEGF-A binds and activates VEGFR2, VEGF-C ${ }^{\mathrm{C} 156 \mathrm{~S}}$ binds and activates VEGFR3, and VEGF-C activates both VEGFR2 and VEGFR3 [40]. After $1 \mathrm{~h}$, VEGF-A and VEGF-C significantly induced Dll4 transcripts, which correlated with an increase in Heyl, Hey2, and Hesl transcripts (Fig. 4a, b). VEGF-C or VEGF-C ${ }^{\mathrm{C} 156 \mathrm{~S}}$ both induced Dll4 expression, as well as the Notch effector, Hes 1 (Fig. 4a, b). Notchl was modestly induced by VEGFA only, while Notch4 expression was unaffected (Fig. 4a).

To determine if VEGF-A and VEGF-C differentially regulate Notch signaling in BECs and LECs, HdLECs and HUVEC were serum starved and treated with either VEGFA or VEGF-C. After 5 h, VEGF-A only induced Dll4, 
A
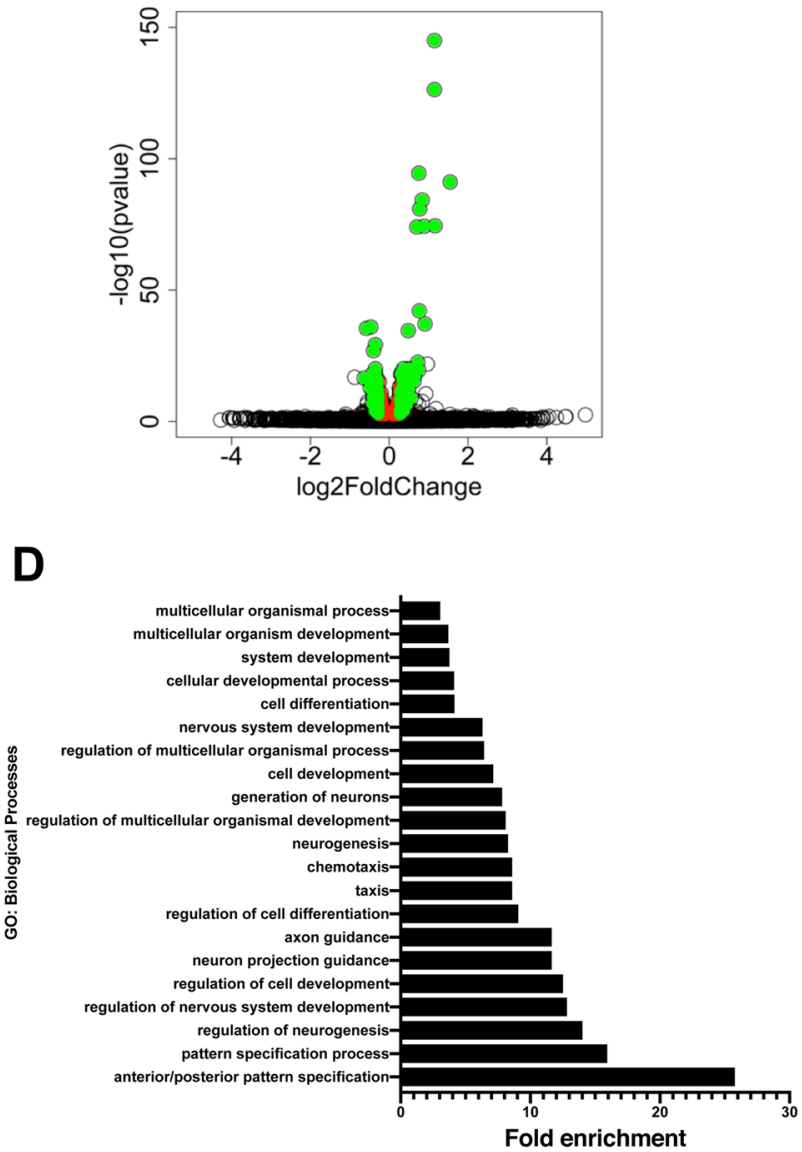

$\mathbf{E}$

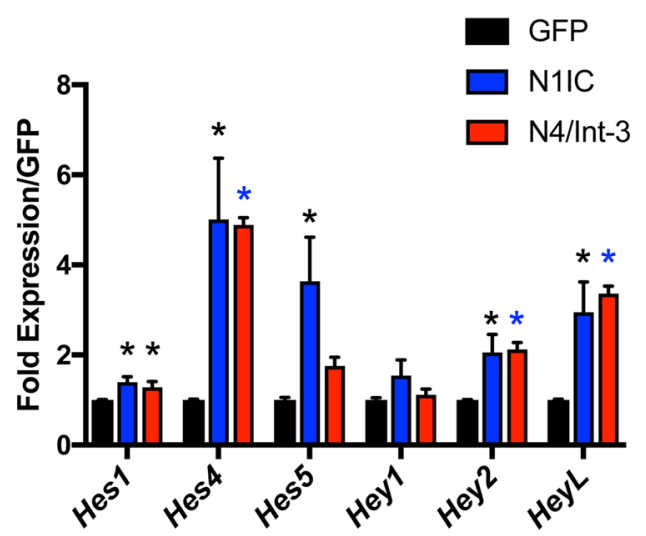

Fig. 3 DLL4/Notch signaling regulated Notch and lymphangiogenic genes via Notch1 and Notch4. HdLECs were seeded on either DLL4FC- or FC-coated plates, and RNA isolated after $6 \mathrm{~h}$, followed by mRNA sequencing. Experiment was performed in triplicate. a Volcano plot of genes downregulated and upregulated by DLL4FC relative to FC controls. b Top 30 genes upregulated and c downregulated by DLL4FC in HdLECs. b, $\mathbf{c} *$ mark Notch pathway and blue asterisks indicate lymphangiogenic genes. d Top GO pathways for biological processes for DLL4-induced genes. e qRT-PCR for
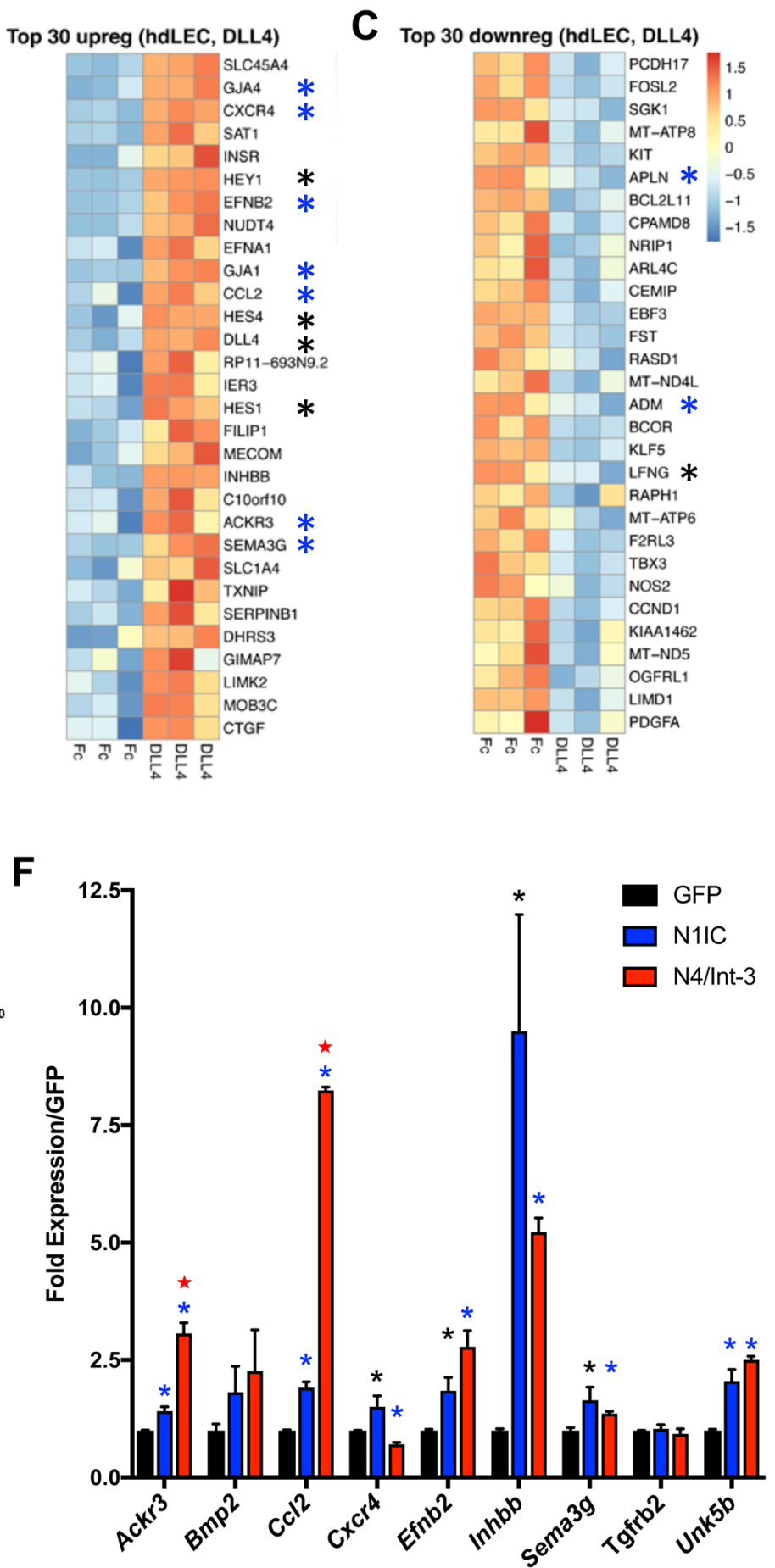

direct targets of Notch signaling and $\mathbf{f}$ lymphangiogenic genes significantly induced in the DLL4-HdLEC assay in HdLECs expressing GFP, N1IC, or N4/Int-3. Data presented for two independent transductions done in duplicate and gene expression determined by delta $\mathrm{CT}$ method and presented relative GFP controls \pm s.e.m. One-way ANOVA performed and significance determined by unpaired $t$ test. e, f $* p<0.05$ or blue asterisks indicate $p<0.003$ N4/Int-3 or N1IC relative to GFP controls. Red stars indicate $p<0.0003$ N4/Int 3 relative to N1IC 
A

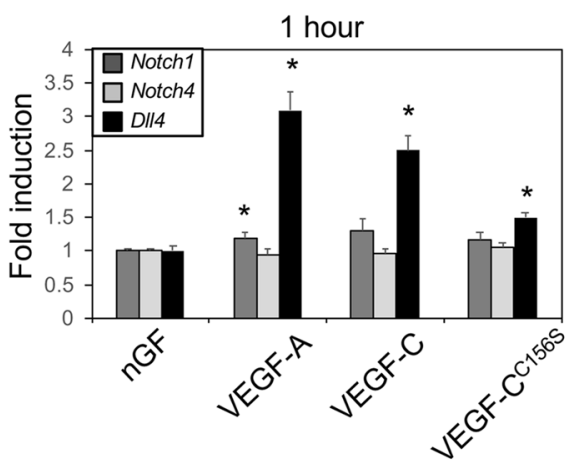

C

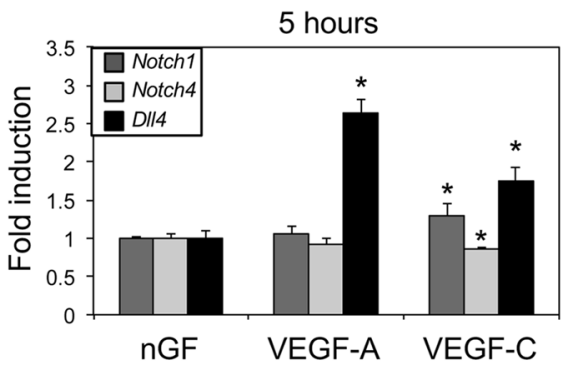

E

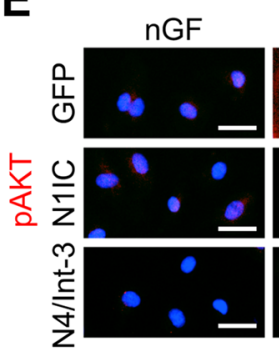

G
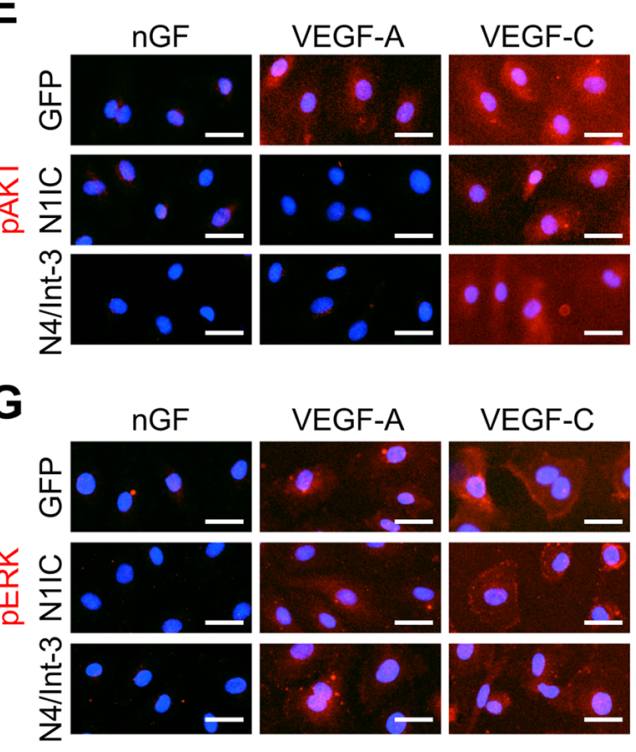

Fig. 4 VEGF and NOTCH signaling modulated each other in LECs. a Notch1, Notch4, and Dll4 expression, and b Heyl, Hey2, and Hes 1 expression determined by qRT-PCR of HdLECs treated for $1 \mathrm{~h}$ with no growth factor (nGF), VEGF-A, VEGF-C, or VEGF-C ${ }^{\mathrm{C} 156 \mathrm{~S}}$. Experiment done in triplicate. Data presented as mean fold induction relative to HdLECs treated with no growth factor \pm s.e.m. $* p<$ 0.05 HdLEC treated with VEGFs relative to HdLEC treated with no growth factor. c Notch1, Notch4, and Dll4 expression, and d Heyl, Hey2, and Hes 1 expression determined by qRT-PCR of HdLECs treated for $5 \mathrm{~h}$ with nGF, VEGF-A, or VEGF-C. Data presented as fold induction relative to $\mathrm{nGF} \pm$ s.d. ${ }^{*} p<0.05$ VEGF-treated HdLEC relative to HdLEC treated with no growth factor. $\mathbf{e}-\mathbf{h}$ GFP-,
$\mathbf{F}$

D
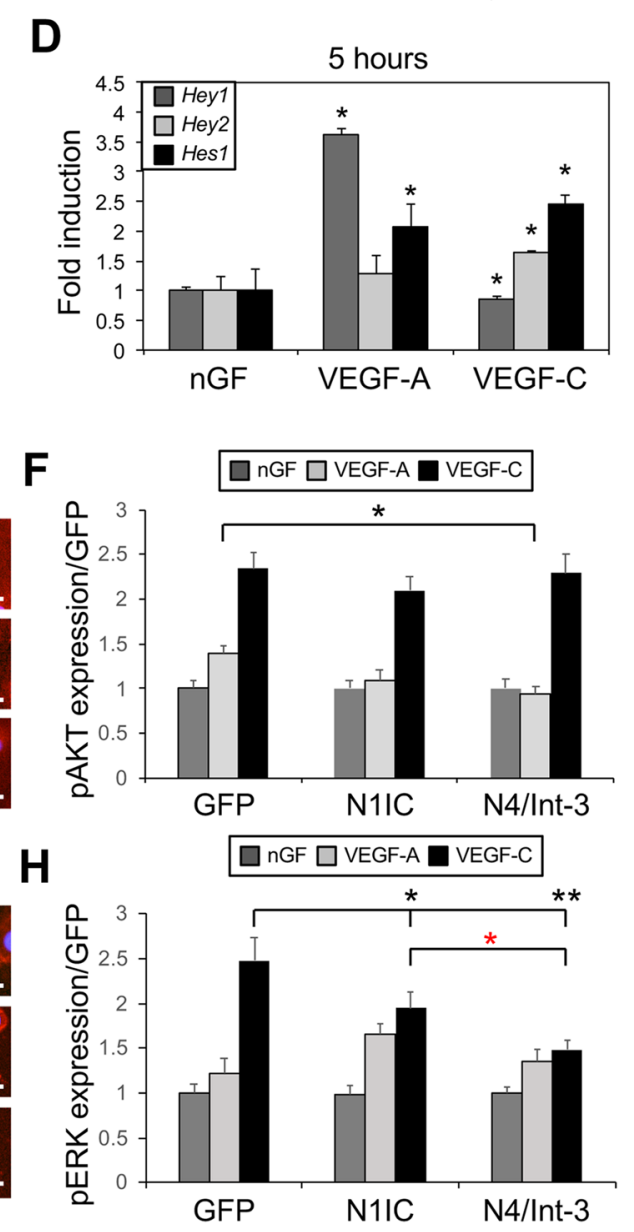

$\mathbf{H}$
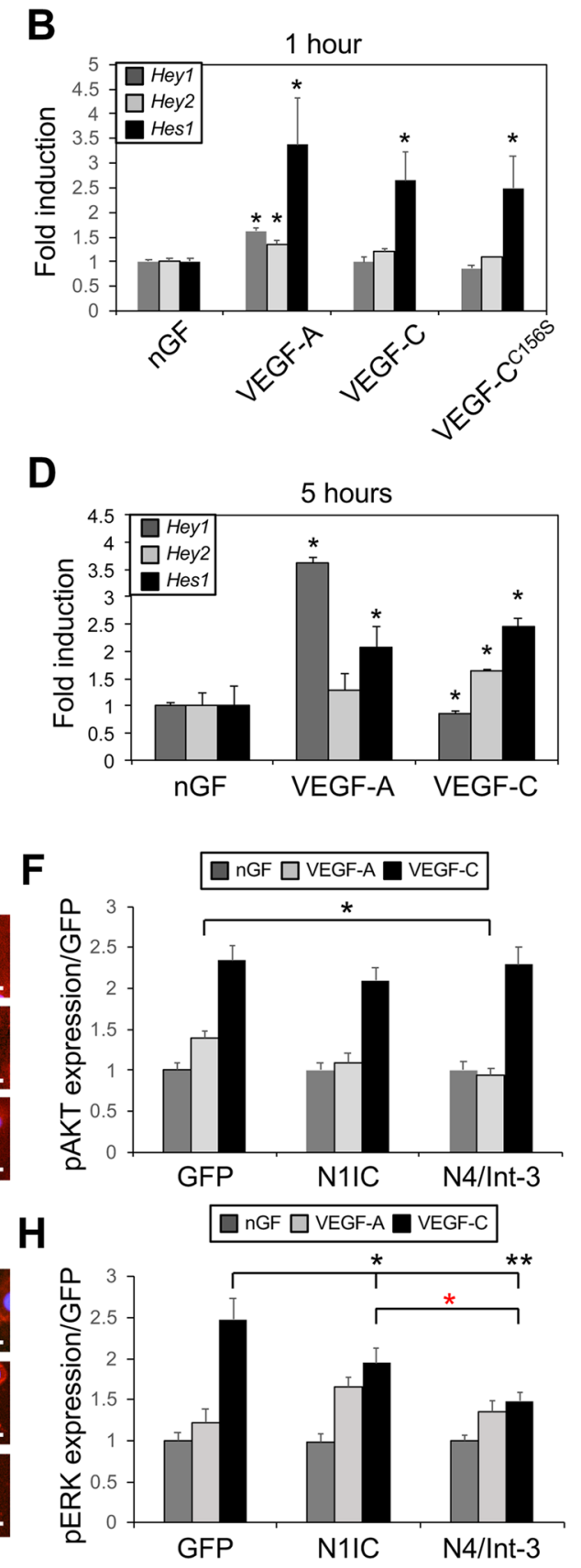

N1IC-, and N4/Int-3-HdLECS were treated with VEGF-A or VEGF$\mathrm{C}$ for $20 \mathrm{~min}$ and then stained for either e phospho-AKT (pAKT) or $\mathbf{g}$ phospho-ERK (pERK). Scale bars, $25 \mu \mathrm{m}$. Quantification of mean, $\mathbf{f}$ pAKT and $\mathbf{h}$ pERK expression normalized by area. Mean data presented for two independent transductions and experiment performed in duplicate. Data presented as fold expression relative to GFP-expressing HdLECs treated with $\mathrm{nGF} \pm$ s.e.m. f pAKT: twoway ANOVA: $p<0.0001$, Dunnett's multiple comparison test $* p<$ 0.002 VEGF-A-treated N4/Int-3 vs. GFP HdLEC. h pERK: two-way ANOVA: $p<0.001$, Dunnett's multiple comparison test $* p<0.03$, $* * p<0.0001$ VEGF-C-treated N1IC or N4/Int-3 vs. GFP HdLEC. $* p<0.003$ VEGF-C-treated N4/Int-3 vs. N1IC HdLEC 
which was associated with an increase in Heyl and Hesl in HdLECs (Fig. 4c, d). VEGF-C induced Dll4 in HdLECs (Fig. 4c), which correlated with an increase in Notchl transcripts (Fig. 4c), as well as Hey2 and Hesl induction (Fig. 4d). In HUVEC, VEGF-A induced Dll4 and Notchl expression, whereas VEGF-C modestly decreased Dll4, Notchl, and Notch4 transcripts (Fig. S4a). Thus, VEGF-A and VEGF-C dynamically and temporally induced Notch activity and specific Notch effectors via induction of Dll4 in cultured LECs.

To determine if Notch 1 or Notch 4 activation altered signaling downstream of the VEGFs, HdLECs expressing either N1IC, N4/Int-3 or GFP were serum starved overnight and then stimulated with VEGF-A or VEGF-C. After 20 min, HdLECs were assessed for AKT and ERK activation by immunofluorescent staining for phospho-AKT and phosphoERK (Fig. 4e-h.). As compared to the GFP controls, activation of AKT was reduced in N4/Int-3 HdLECs treated with VEGF-A. Activation of AKT by VEGF-C was unaffected in HdLECs expressing either N1IC or N4/Int-3. Levels of ERK activity were unaffected by constitutive activation of Notch1 or Notch4 in all VEGF-A-treated HdLECs (Fig. 4e, f), whereas ERK activation was reduced in N1IC and N4/ Int-3 HdLECs treated with VEGF-C relative to controls (Fig. 4 g, h). Previous studies have shown that Notch signaling alters the expression of the VEGF-A and VEGF-C receptors, VEGFR2 and VEGFR3 [9, 12, 18, 41]. The reduction in AKT and ERK activation downstream of VEGFs may be secondary to Notch signaling effects on VEGFR expression. Therefore, the expression of Vegfr 2 and Vegfr 3 was determined in HdLECs with N1IC and N4/Int-3 by qRT-PCR. Both N1IC and N4/Int-3 downregulated Vegfr2, while they induced Vegfr3 (Fig. S4b). Taken together, the data suggests that the decrease in AKT activation by VEGF-A and ERK activation by VEGF-C may be due to reduced VEGFR2 levels, and not VEGFR3.

\section{Embryonic dermal lymphangiogenic defects in Notch4 mutant mice}

A prior report has shown that loss of LEC Notchl in embryos leads to increased LEC proliferation and tip cells [14]. To determine the role of Notch4 in embryonic lymphangiogenesis, we evaluated $\mathrm{Notch}^{-/-}$mice and compared their lymphatic phenotype to that of $\mathrm{Notch}^{+/-}$and wild-type littermates. To confirm that NOTCH4 protein is absent in the $\mathrm{Notch}^{-/-}$embryos, western blots using lysates collected from E14.5 embryos and staining of P4 dermal tissue with an antibody against the intracellular domain of NOTCH4 were performed. As compared to wild-type littermates, NOTCH4 expression was absent in Notch4 ${ }^{-/-}$tissues (Fig. $\mathrm{S} 5 \mathrm{a}-\mathrm{c})$. NOTCH1 expression determined by immunostaining of P4 dermis was unaffected (Fig. S5d).
Analysis of E14.5 Notch $^{-/-}$dermal wholemounts revealed that the distance between the two migrating lymphatic fronts was decreased relative to wild-type and Notch $^{+/-}$littermates (Fig. 5a, b). This correlated with an increase in the number of lymphatic fronts migrating toward the midline in the Notch $4^{-/-}$dermis (Fig. 5c). Further analysis of the lymphangiogenic sprouts at the migration front revealed that the length from the front to the first branchpoint did not differ between mutants and controls (Fig. 5d). We next evaluated the lymphatic vessel caliber at the lymphangiogenic front and in the maturing plexus. The caliber of the vessel adjacent to the first branch-point at the front did not differ between mutant and control mice (Fig. 5e, f). However, a significant reduction of vessel caliber was observed in the maturing lymphatic plexus of Notch $^{-/-}$. Although the lymphatic vessel diameter was reduced in the maturing Notch $4^{-/}$plexus, branching was similar between mutants and controls (Fig. S6a). To determine if the reduced dermal lymphatic vessel caliber was due to a change in LEC proliferation, wild-type and Notch $^{-/-}$E14.5 dermal skin were stained for the proliferation marker, KI67, and LYVE1. Dermal LEC proliferation was similar between $\mathrm{Notch}^{-/-}$and control mice (Fig. S6b, c). As NOTCH4 is also expressed by the blood vasculature [20,24], we evaluated the underlying dermal blood vascular network in E14.5 Notch4 $^{-/-}$and Notch $4^{+/-}$embryos. Consistent with prior studies, the density and branching of the blood vasculature were unaffected in the Notch4 nulls (Fig. S7) [24, 42].

We next evaluated the dorsal dermal lymphatic phenotype at E16.5 in wild-type and Notch4 ${ }^{-/-}$embryos. The lymphatic fronts had reached the midline and merged in both wild-type and Notch $^{-/-}$embryos. Analysis of the lymphatic plexus revealed that it was disorganized with an increase in the distance between branch-points, while there was no difference in the mean vessel caliber in Notch $^{-/-}$dermis relative to controls (Fig. $5 \mathrm{~g}-\mathrm{i}$ ). Lymphatic valves were observed in both mutants and controls. A recent study has shown that reduced branching in the dermal lymphatic plexus was associated with an increase in blunt-end sprouts due to reduced VEGFR3 signaling, which in turn led to a less branched network [43]. In contrast, increased VEGFR3 signaling was associated with reduced blunt-ended sprouts and a more densely branched network [44]. Since we observed a decrease in the dermal lymphatic branching in the E16.5 Notch $4^{-1-}$ embryos, we assessed the sprout phenotype at E14.5 during active lymphangiogenesis. The sprouts of control embryos uniformly expressed LYVE1 and were elongated with numerous filopodia consistent with a lymphangiogenic phenotype (Fig. S8). In contrast, LECs in sprouts in the $\mathrm{Notch}^{-/-}$lymphatic vasculature were often rounded with reduced and blunted filopodia.

Thus, Notch4 mutant mice had a distinct dermal lymphatic phenotype from that observed in mice with LEC 
A

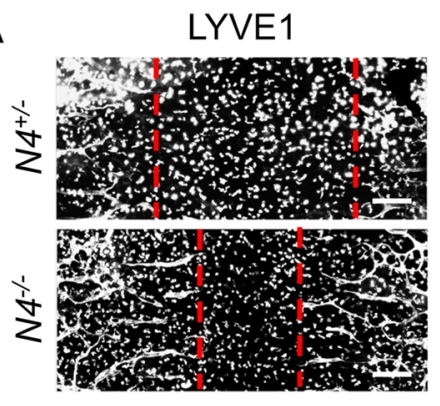

C

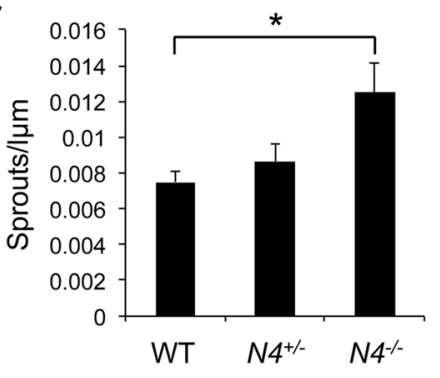

E

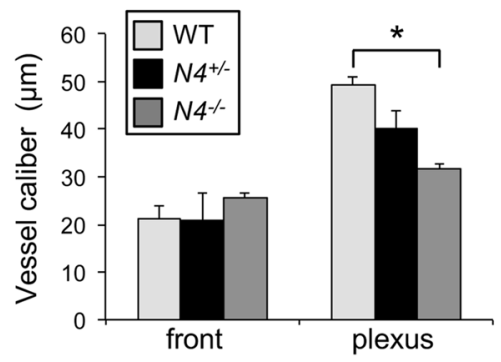

G

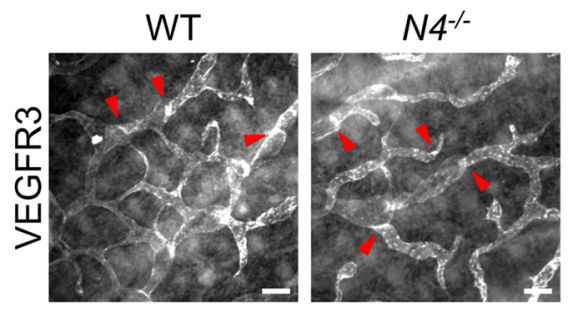

B

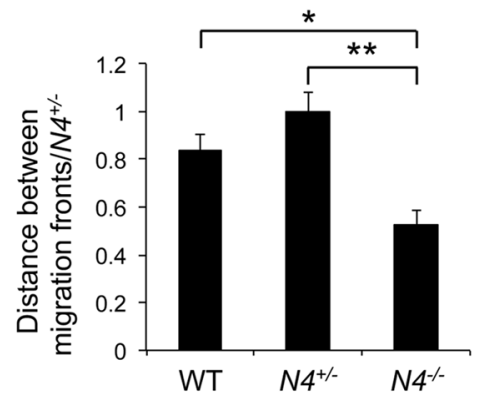

D

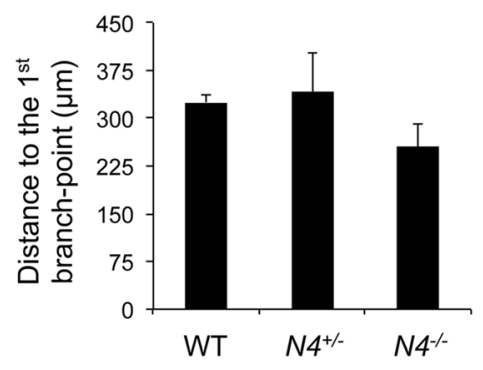

$\mathbf{F}$

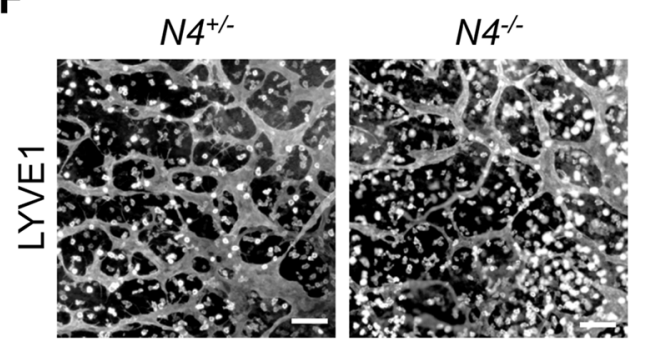

H

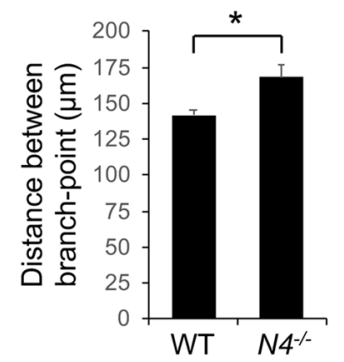

I

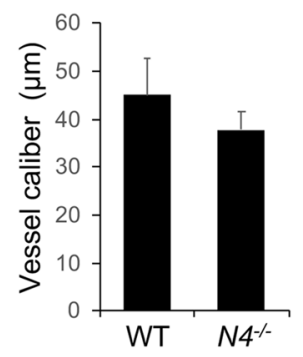

Fig. 5 Loss of Notch4 altered embryonic dermal lymphangiogenesis. a-f Dermal lymphatic phenotype was determined for E14.5 Notch $^{-/-}$ $\left(\mathrm{N}^{-/-}\right)$, Notch4 $4^{+--}\left(\mathrm{NH}^{+/-}\right)$and wild-type (WT) wholemounts stained for LYVE1. a Representative image of $\mathrm{N4}^{-/-}$and $\mathrm{N}^{+/-}$dermal wholemounts. Red dotted line marks leading edge of lymphangiogenic front. Scale bars, $100 \mu \mathrm{m}$. b Quantification of the distance between migration fronts, normalized to the $\mathrm{N4}^{+/-}$controls. Data presented \pm s.e.m. One-way ANOVA: $p=0.014, t$ test: $* p<3 \times 10^{-4}, * * p<$ 0.001 . wt $(n=5), N 4^{+-}(n=13), N 4^{-/} \quad(n=4)$. c Quantification of the number of lymphangiogenic sprouts normalized by length of the front. Data presented \pm s.e.m. One-way ANOVA: $p=0.023$, $t$ test: $* p<0.02$. WT $(n=6), N 4^{+/-}(n=11), N 4^{-/}(n=6)$. d Quantification of the sprout length from the migration front to first branch-point. Data presented \pm s.e.m. WT $(n=7), N 4^{+/-}(n=8)$, $N 4^{-1-}(n=6)$. e Quantification of the average vessel caliber at the lymphangiogenic front and in the maturing plexus. Data presented \pm s.e.m. One-way ANOVA: $p=0.019, t$ test: $* p<0.03$, front analysis- WT $(n=7), N 4^{+-}(n=13), N 4^{-/-}(n=7)$, plexus analysis - WT $(n=6), N 4^{+/-}(n=9), N 4^{-/-}(n=8)$. f Representative image of the E14.5 maturing lymphatic plexus in $\mathrm{N}^{-/-}$and $\mathrm{N}^{+/-}$littermates. Scale bars, $100 \mu \mathrm{m}$. $\mathbf{g}$-i Dermal lymphatic phenotype determined for E16.5 Notch4 $^{-/-}\left(\mathrm{N}^{-/-}\right)$and wild-type (WT) wholemounts stained for VEGFR3. g Representative image of the E16.5 dermal lymphatic plexus in $\mathrm{N4}^{-/-}$and WT littermates. Red arrowheads mark lymphatic valves. Scale bars, $100 \mu \mathrm{m} ; \mathbf{h}$ quantification of mean distance between branch-points. Data presented \pm s.e.m. $t$ test: $* p<0.03$ WT $(n=4), N 4^{-/-}(n=6)$. i Quantification of the average vessel caliber of the dermal lymphatic plexus at E16.5. Data presented \pm s.e.m. WT $(n=6), N 4^{-/-}(n=8)$ 
Fig. 6 Notch signaling inhibited LEC migration. a Confluent N1IC-, N4/Int-3-, Hey1-, Hey2-, or GFP-expressing HdLECs were scratched and representative images for 0 and $25 \mathrm{~h}$ presented. Scale bars, $2.5 \mu \mathrm{m}$. b Quantification of percent open wound area at 0,8 , and $25 \mathrm{~h}$. Data presented \pm s.e.m. two-way-ANOVA: $p<0.0012, t$ test: ${ }^{*} p<0.002$ N1IC or N4/Int-3 vs. GFP at 8 and $25 \mathrm{~h}, * p<0.002 \mathrm{~N} 4 /$ Int-3 vs. N1IC at 25 h. c Confluent N1IC-, N4/Int-3-, or GFP-expressing HdLECs were treated with mitomycin $\mathrm{C}$ and scratched and representative images for 0 and $24 \mathrm{~h}$ presented. Scale bars, $100 \mu \mathrm{m}$. d Quantification of percent open wound area at $0,4,8$, and $24 \mathrm{~h}$. Data presented \pm s.e.m. two-way ANOVA: $p<0.0001$, Dunnett's multiple comparison test $* p<$ 0.0001 N1IC or N4/Int-3 vs. GFP. $t$ test: ${ }^{*} p<0.0001 \mathrm{~N} 4 /$ Int-3 vs. N1IC
A

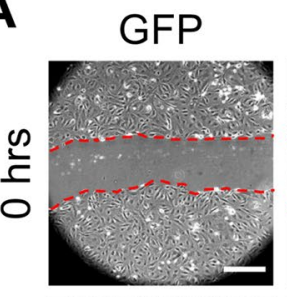
N1IC

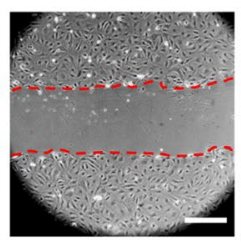

N4/Int-3
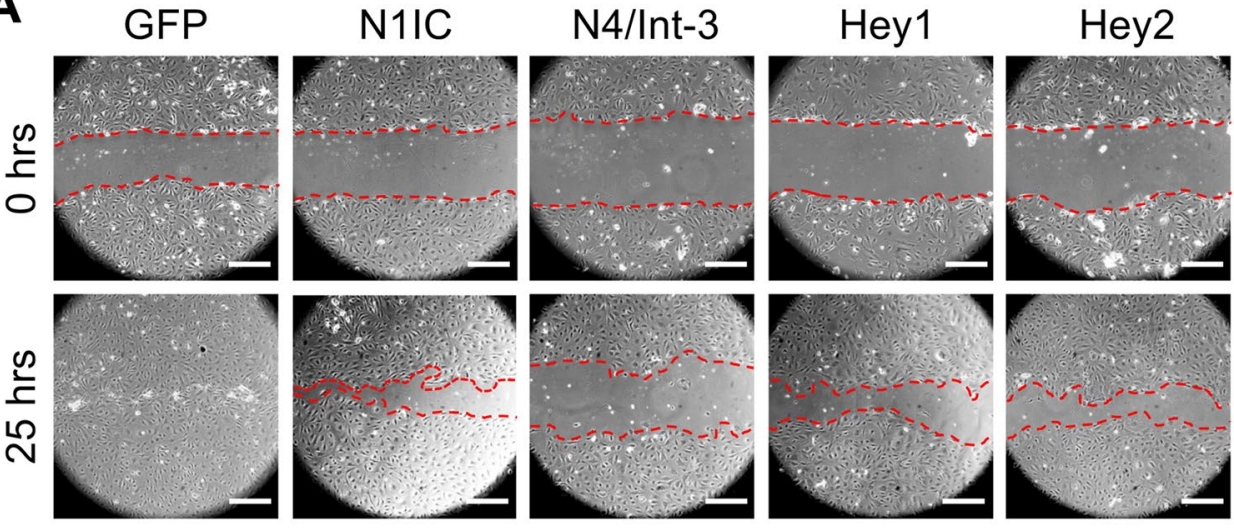

B
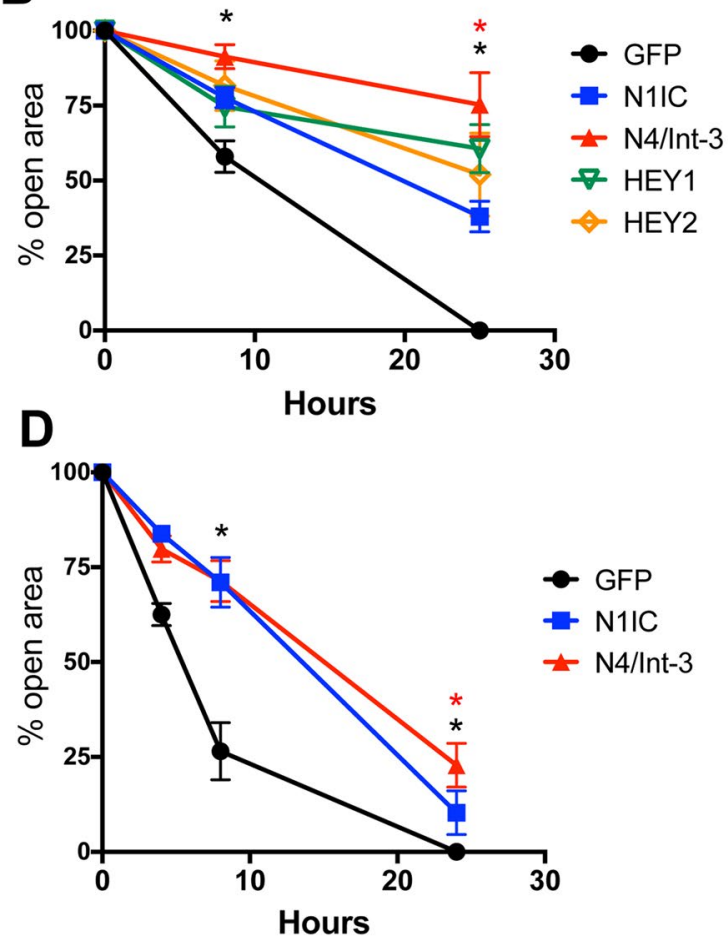

$24 \mathrm{hrs}$
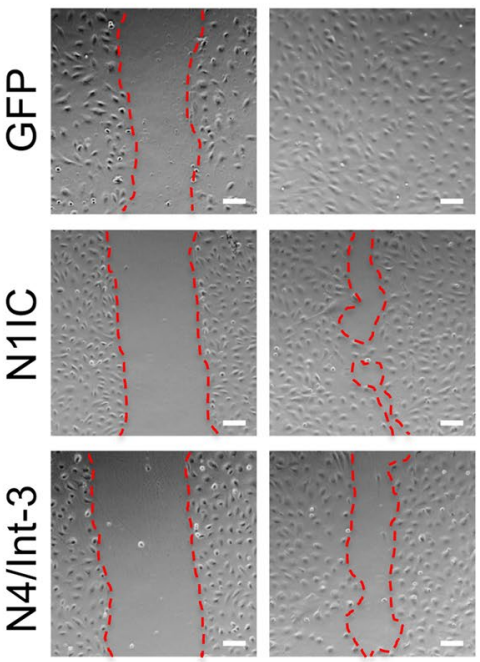

Notchl deletion [14]. Rather than increased vessel diameter due to increased proliferation and branching due to increased sprouting lymphangiogenesis, the embryonic dermal lymphatics in Notch4 nulls had increased front closure, an early decrease in the lymphatic vessel caliber, and reduced branching without a change in LEC proliferation.

\section{NOTCH4 activation preferentially inhibited LEC migration}

To determine the effects of Notch 1 and Notch 4 signal activation on LEC migration, a monolayer-wounding assay was performed using HdLECs expressing either N1IC or N4/Int-3. Relative to control GFP-expressing HdLEC, both
N1IC and N4/Int-3 expression inhibited LEC migration (Fig. 6a, b). We next evaluated the effect of overexpressing the downstream Notch effectors HEY1 and HEY2 on HdLEC migration (Fig. 6a, b). Ectopic expression of HEY1 and HEY2 suppressed migration relative to control HdLECs. Further analysis revealed that N4/Int-3 was a significantly stronger inhibitor of LEC migration than $\mathrm{N} 1 \mathrm{IC}$ at $25 \mathrm{~h}$. To insure the difference in migration was not due to changes in LEC proliferation, N1IC-, N4/Int3- and GFP-expressing HdLECs were treated with mitomycin $\mathrm{C}$ and LEC migration determined. Similar to the initial migration assay, both N1IC and N4/Int-3 suppressed HdLEC migration with N4/Int-3 suppressing migration significantly more than N1IC at $24 \mathrm{~h}$ (Fig. 6c, d). 
Inhibition of lymphatic endothelial canonical Notch signaling increased dermal lymphatic vessel density

Notch4 has been shown to signal via RBPjк-dependent (canonical) and RBPjк-independent (non-canonical) downstream pathways [45-47]. To determine the effects of LEC specific loss of canonical Notch signaling on dermal lymphangiogenesis, we used the inducible ProxlCreER ${ }^{T 2}$ driver to induce expression of a DNMAML transgene [26]. $D N M A M L$ encodes a dominant negative form of Mammalian Mastermind-like 1 (MAML1) that binds the NOTCH/RBPjк complex to form an inactive complex and blocks the recruitment of transcriptional co-activators. ProxlCreER ${ }^{T 2}$ mice were crossed with $D N M A M L^{f / f l}$ mice to generate ProxlCreE $R^{T 2} ; D N M A M L^{f / /}$ embryos $\left(D N M A M L^{L E C}\right)$ and $D N M A M L^{f /+}$ control littermates. To circumvent effects on early lymphatic specification caused by loss of Notch signaling in LECs [18], tamoxifen was administered to pregnant females at E12.5, just as sprouting lymphangiogenesis begins, and the dermal lymphatic phenotype analyzed at E14.5. Unlike the Notch4 nulls, the closure of the migration fronts was the same between DNMAML ${ }^{L E C}$ and control (Fig. 7a, b). The number of sprouts along the migrating front and the length of the sprout to the first branch-point were similar between mutants and controls (Fig. 7c, d). In contrast, the lymphatic density was nearly $25 \%$ greater in the DNMAML ${ }^{L E C}$ compared to controls (Fig. 7e). The increase in the DNMAML ${ }^{L E C}$ dermal lymphatic density correlated with an enlargement of the lymphatic vessel caliber at the lymphangiogenic front and in the maturing plexus (Fig. 7f, g). As compared to controls, $D N M A M L^{L E C}$ dermal lymphatics had an increase in the number Prox1+/LYVE1+ LECs (Fig. 7h), while branching in the mature plexus was unaffected (Fig. 7i). The increase in vascular density was specific to the lymphatics as blood vessel density was unchanged in DNMAML ${ }^{L E C}$ mutants (Fig. S9). Thus, we found that inhibition of RBPjк-dependent Notch signaling resulted in increased lymphatic vessel density and caliber associated with an increase in LECs, suggesting that canonical Notch signaling suppressed LEC proliferation in the embryonic dermal lymphatics.

\section{Notch $^{-/-}$and DNMAML ${ }^{\text {LEC }}$ embryos display distinct lymphatic phenotypes at E17.5}

To assess the functionality and patterning of the dermal lymphatics, lymphangiographies were performed on E17.5 Notch $^{-/-}, D N M A M L^{L E C}$, and control littermates. Dye was injected within the dermis in the periorbital region and uptake by the lymphatics assessed after $1 \mathrm{~min}$. The Notch ${ }^{-/-}$lymphatic plexus had reduced branching with tortuous vessels relative to the more uniform lymphatics of controls (Fig. 8a). One of the $8 \mathrm{Notch}^{-/-}$embryos analyzed had blood-filled dermal lymphatics at E17.5 (Fig. 8b).
In contrast to the $\mathrm{Notch}^{-/-}$phenotype, DNMAML ${ }^{L E C}$ dermal lymphatics were dilated relative to control littermates (Fig. 8c). One out of $5 D N M A M L^{L E C}$ embryo lymphatics was leaky (Fig. 8d), which was not observed in controls or Notch $^{-/-}$lymphangiographies. These data demonstrate that the Notch4 null and mice with a loss of LEC RBPjкdependent Notch signaling have distinct phenotypes at E17.5, as well as at E14.5 (Figs. 5 and 7).

\section{Canonical Notch signaling is unaffected in Notch4 ${ }^{-/-}$ embryonic dermal lymphangiogenesis}

As we observed a difference between the embryonic dermal lymphatic phenotypes of $\mathrm{Notch}^{-/-}$and DNMAML ${ }^{L E C}$ mutants, we evaluated canonical Notch signaling by introducing the NVR and ProxTom alleles into the Notch4 null background. Loss of Notch4 did not change canonical Notch signaling at the lymphangiogenic vascular front, nor the maturing lymphatic plexus (Fig. 9, S6d). This data suggested that Notch4 is not necessary for canonical Notch signaling in the embryonic dermal lymphatics.

\section{Discussion}

NOTCH1 and NOTCH4 are expressed and Notch signaling active in the embryonic and early postnatal dermal lymphatic vasculature $[12,14]$, suggesting a role for both Notch proteins in embryonic lymphangiogenesis. Loss of Notch4 was shown to exacerbate the Notch1 null embryonic blood vascular phenotype, suggesting that Notch 1 and Notch 4 have overlapping functions in the blood endothelium [24]. In contrast to the blood endothelium, we found that loss of Notch4 led to a distinct embryonic dermal lymphangiogenic phenotype, than that observed in mice with LEC deletion of Notchl [14], or inhibition of canonical Notch signaling, presented here. At E14.5, Notch4 null embryos displayed an increase in the closure of the lymphangiogenic fronts to the midline, reduced vessel caliber in the maturing plexus, and an increase in blunt-ended sprouts, while LEC proliferation was unaffected. By E16.5, the dermal lymphatic plexus in Notch $4^{-/-}$has reduced branching and tortuous lymphatic vessels, which may be secondary to the increase in bluntended sprouts at E14.5. In cultured LECs, constitutive activation of Notch 4 was a stronger inhibitor of migration than Notch1 activation and induced a subset of lymphangiogenic genes. In contrast, loss of LEC Notch1 at E10.5 increased embryonic dermal lymphatic density, due to increased LEC proliferation and decreased LEC apoptosis [14]. Similar to the Notch1 LEC knockout, we demonstrate that LEC expression of DNMAML, which inhibits canonical Notch/ RbpJ $x$ signaling, increased the dermal lymphatic vascular density consistent with an increase in LEC proliferation and 


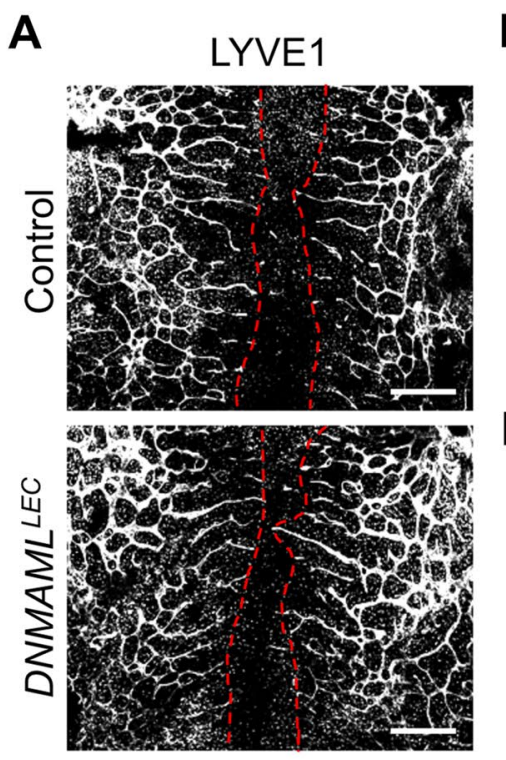

$\mathbf{F}$
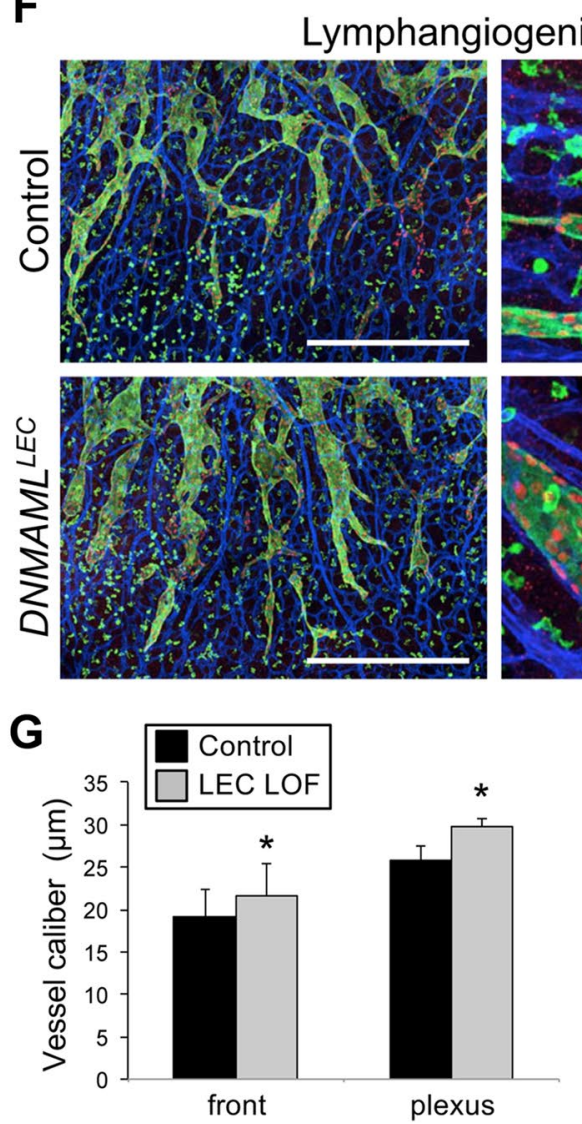

B
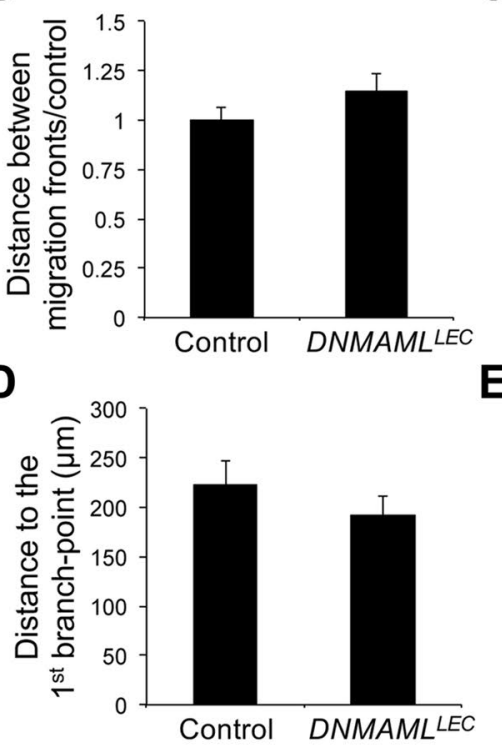

C

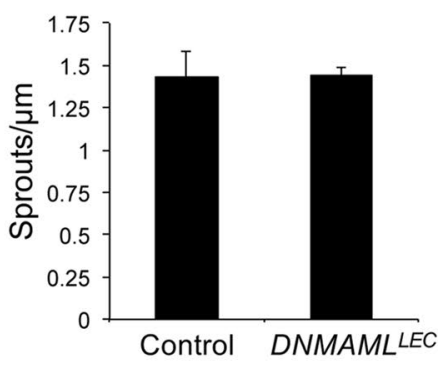

E

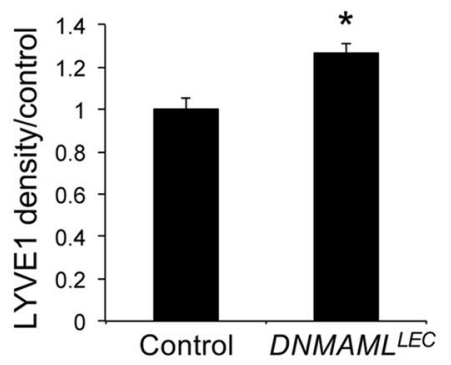

Maturing plexus
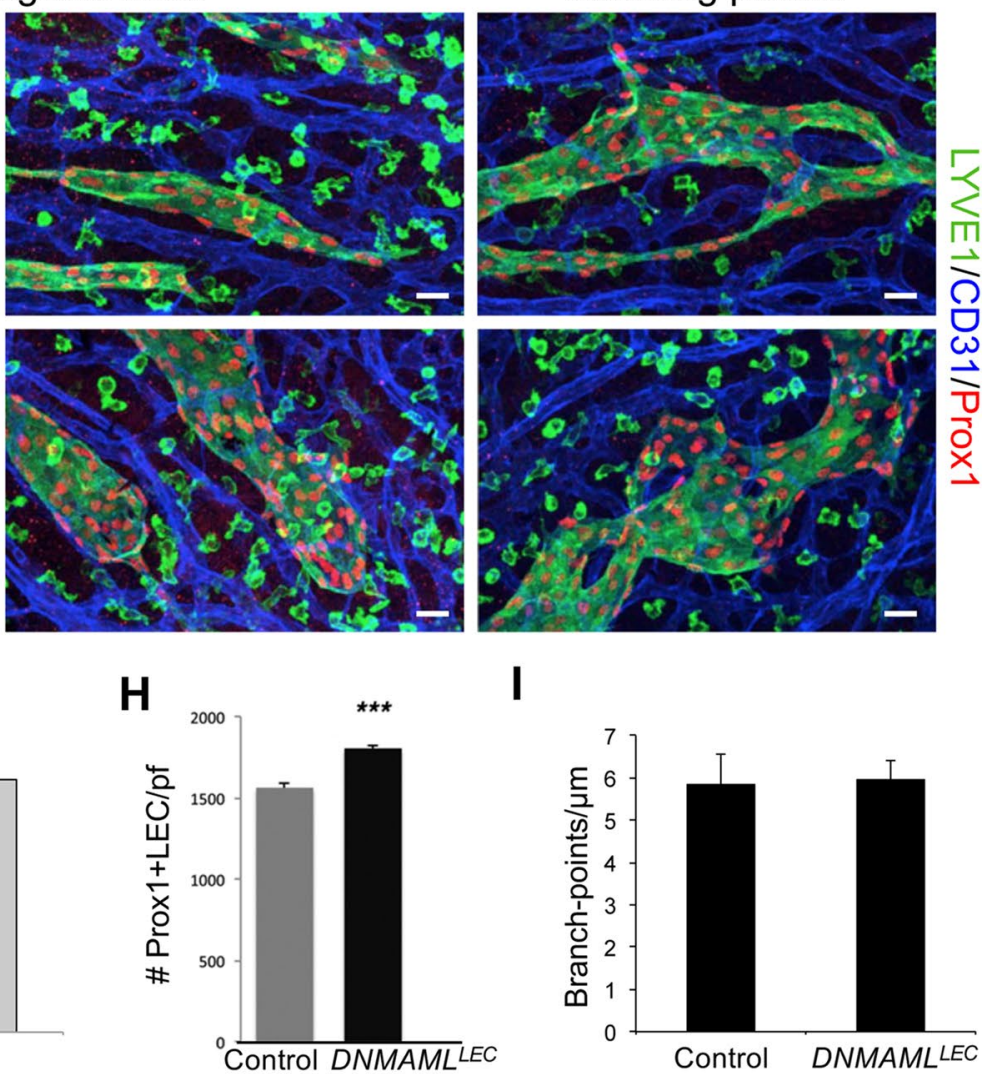

I

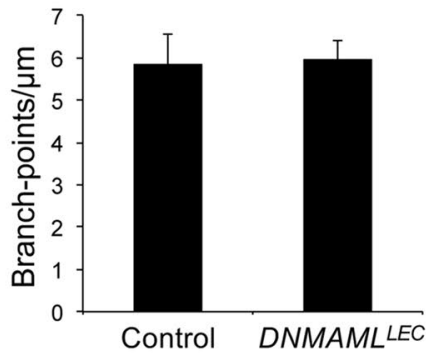

viability. Distinct functions for Notch1 and Notch4 have been described for endothelial progenitor cells, where Dll4 signaling via Notch4 specifically induced EphrinB2 and increased proliferation and migration of cultured cells [48]. More recently, it was proposed that endothelial Dll4/Notch1 signaling induces Hey 2 to suppress proliferation and tip cell formation, while Jag1 activates Notch4 to induce Hey1 and promote vessel maturation while having no effect on vascular density [49]. Taken together, we propose that Notch1 and Notch4 signal dynamically to regulate lymphangiogenesis and control migration and branching, versus proliferation and cell viability by distinct mechanisms.

Our studies suggest that Dl14 signaling via Notch1 and Notch 4 have overlapping and unique transcriptional targets 
४Fig. 7 Loss of canonical Notch signaling in LECs increased dermal lymphatic density. ProxlCreER ${ }^{T 2}$ and $D N M A M L^{f / f l}$ mice were crossed, tamoxifen administered at E12.5 and dorsal dermis analyzed at E14.5. a Representative images of LYVE1 staining of ProxlCre $E R^{T 2} ; D N M A M L^{f l /+}\left(D N M A M L^{L E C}\right)$ and DNMAML $L^{f /+}$ (control) dermis. Red dotted line marks leading edge of lymphatic fronts. Scale bars, $1000 \mu \mathrm{m}$. b Quantification of the distance between migration fronts, normalized to the $D N M A M L^{\mathrm{fl} /}+$ controls. Data presented \pm s.e.m. Control $(n=7)$, DNMAML $L^{L E C}(n=9)$. c Quantification of the number of lymphangiogenic sprouts normalized by length of the front. Data presented \pm s.e.m. Control $(n=8), D N M A M L^{L E C}$ $(n=6)$. d Quantification of the distance between migration fronts, normalized to the $D N M A M L^{\mathrm{f} /}+$ controls. Data presented \pm s.e.m. Control $(n=8), D N M A M L^{L E C}(n=6)$. e Quantification of average LYVE1+ vessel density normalized by area. Data presented relative to control \pm s.e.m. $t$ test $* p<0.002$, control $(n=7), D N M A M L$ LEC ( $n=9)$. f LYVE1, CD31, and PROX1 staining of DNMAML ${ }^{L E C}$ mutant and control dermal wholemounts. Images represent low (left) and high (middle) magnification of the lymphangiogenic front and the maturing plexus (right). Scale bars, $500 \mu \mathrm{m}$ (left), $100 \mu \mathrm{m}$ (middle, right). g Quantification of the average vessel caliber at the lymphangiogenic front and in the maturing plexus. Data presented \pm s.e.m. $t$ test: $* p<0.04,{ }^{*} p<00.01$, Control $(n=8), D N M A M L^{L E C}$ $(n=6)$. h Quantification of the number of PROX1+/LYVE1+ LECs per field (pf). Data presented as \pm s.d. $* * * p<0.001$. Control $(n=8)$, $D N M A M L^{L E C}(n=6)$. i) Quantification of the average number of branch-points normalized to unit of vessel length. Data presented \pm s.e.m. control $(n=3), D N M A M L^{L E C}(n=5)$

in HdLECs. Constitutive Notch1 and Notch4 activation in LECs both induced the expression of Notch effectors (Hes 1, Hes4, Hey1, Hey2, HeyL) and lymphangiogenic genes, such as EphrinB2, and $C x 37$ [50], as well as downregulated essential genes in lymphangiogenesis, Podoplanin, and Proxl. In contrast, Hes 5 expression was induced by N1IC, and not N4/Int-3. DLL4/Notch-induced genes involved in chemokine signaling were also differentially regulated by Notch1 and Notch4. Expression of Cxcr4 was induced by Notch 1 activation, but suppressed by Notch 4 signaling. In LECs, CXCR4 signaling promoted woundinduced and VEGF-C driven lymphangiogenesis in vivo, while in vitro it induced chemokine-driven LEC migration $[31,38]$. Thus, it is possible that loss of Notch4 led to an increase in CXCR4 expression, which in turn increased the LEC migration toward the midline. N4/Int-3 was also a significantly stronger inducer of $C c l 2$ and $A c k r 3$, than N1IC. LEC-derived CCL2 has been shown to promote the recruitment of monocytes and macrophages to sites of lymphangiogenesis, where they deliver VEGF-A and VEGF-C [33, 36]. In murine lymphatic development, ACKR 3 functions to suppress LEC growth by scavenging adrenomedullin (ADM) [35]. Interestingly, we found that Dll4/Notch signaling also suppressed Adm expression, suggesting that Dll4/Notch4 signaling suppresses ADM signaling to regulate lymphatic development. While our gene expression studies begin to elucidate some of the mechanisms by which Dll4/Notch1 and Dll4/Notch4 signaling regulates lymphangiogenesis, further studies are necessary to understand the complexity of Notch1 and Notch4 signaling in LECs.

Prior studies have shown that VEGF-C induces DLL4 in LECs leading to Notch activation [10]. We expanded these studies to understand the role of time and specific VEGFRs in this process. Using VEGF-C $\mathrm{C}^{\mathrm{C} 156 \mathrm{~S}}$ which specifically binds VEGFR3 and VEGF-A which binds VEGFR2, we found that VEGFR2 signaling was a stronger and faster inducer of Dll4/Notch signaling than VEGFR3 signaling. We also discovered that the induction of the Hes and Hey genes was time- and VEGF-dependent in HdLECs. VEGF-A induced Hes 1 at $1 \mathrm{~h}$ which persisted until $5 \mathrm{~h}$, while significant Heyl upregulation was not observed until $5 \mathrm{~h}$. In LECs, VEGFA was a stronger inducer of Heyl, while VEGF-C induced Hey2. This differential response of Hes and Hey genes to VEGF-A and VEGF-C was specific to LECs, as VEGF-C had no effect on Dll4, or Notch gene expression in HUVEC. Our studies also suggest that additional Hes and Hey gene family members than those studied in the blood vasculature, Hes 4 and HeyL, have a role in transmitting Notch signaling in LECs.

Our studies also revealed that Notch1 and Notch4 differentially altered signaling downstream of VEGF-A/VEGFR and VEGF-C/VEGFR. Constitutive activation of Notch4 blocked AKT activation by VEGF-A, and ERK activation by VEGF-C, whereas Notch1 activity only modestly suppressed VEGF-C activation of ERK signaling. Together, these data suggest that Notch4 has a role in modulating VEGFR2 and VEGFR3 signaling down the PI3K/AKT and RAS/MAPK pathway.

The dermal lymphatic phenotypes were distinct between Notch $4^{-/-}$and DNMAML ${ }^{L E C}$, suggesting Notch4 signals at least in part via a non-canonical pathway. Notch4 has been shown to signal via canonical (RBPjk-dependent) and noncanonical (RBPjk-independent) Notch pathways in multiple cells types [45-47]. In endothelial cells, Notch4 activation blocked LPS-induced apoptosis via RBPjк-independent upregulation of $\mathrm{Bcl} 2$ [45]. In mice, NOTCH4 activation in the ductal epithelium required RBPjx for physiological alveolar development, but not for breast cancer development, suggesting Notch4 functions via both canonical and noncanonical pathway in the breast endothelium [46, 47]. We observed that canonical Notch signaling was unchanged in the embryonic dermal LECs in Notch4 nulls suggesting that the Notch4 dermal lymphatic phenotype did not occur via a RBPjк-dependent mechanism. However, it is possible that the variable phenotypes are due to differences in the penetrance of global Notch4 loss versus a tamoxifen-induced cell mosaic expression of DNMAML in LECs.

An increase in the closure of the two lymphangiogenic fronts was observed in Notch4 mutants that correlated with reduced vessel caliber in the absence of a change in LEC proliferation. This phenotype is consistent with an increase 
Fig. 8 Loss of Notch4 and deletion of LEC canonical Notch signaling resulted in distinct lymphatic phenotypes at E17.5. a, b Lymphangiography of E17.5 wild-type $(n=6)$ and Notch4 $^{-/-}(n=8)$ embryos. Representative images of wild-

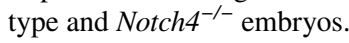
Boxed area enlarged to the right. b Notch $4^{--}$embryos with blood-filled dermal lymphatics (white arrowheads). Boxed area enlarged to the right. c, d ProxlCreER ${ }^{T 2}$ and DNMAML ${ }^{f / f l}$ mice were crossed and tamoxifen administered at E12.5 and lymphangiography performed at E17.5. Control $(n=12)$, DNMAML${ }^{L E C}(n=5)$. c Representative images of DNMAML $L^{f /-}$ control and DNMAML ${ }^{L E C}$ embryos. Boxed area enlarged to the right. d DNMAML ${ }^{L E C}$ embryo with leaking dermal lymphatic vessels (red arrowheads). Boxed area enlarged below
A

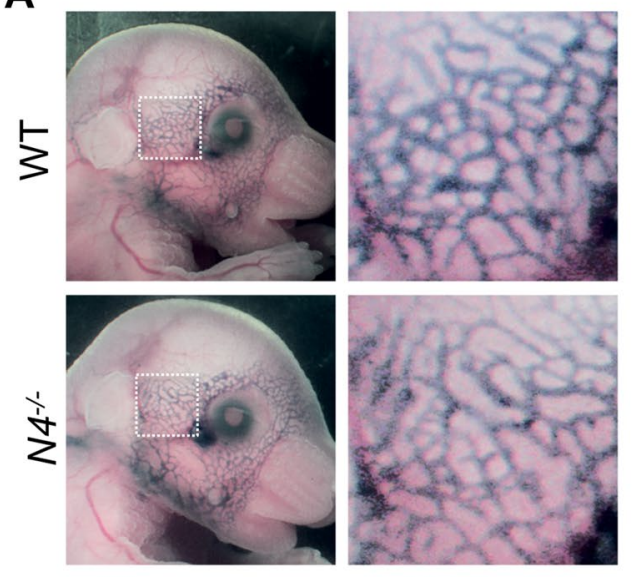

B

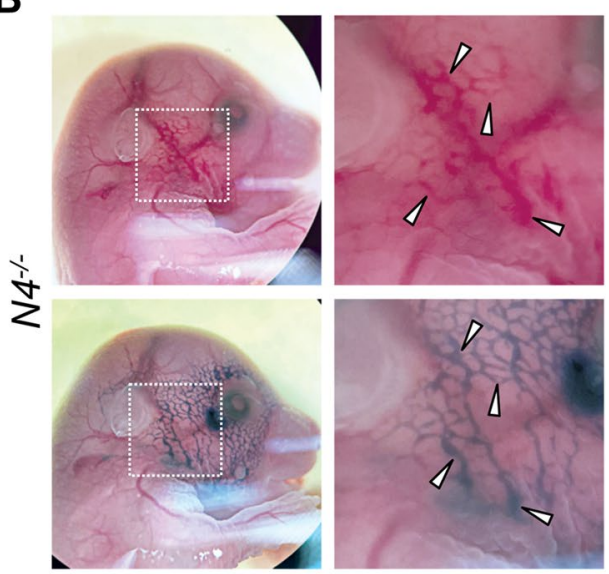

C

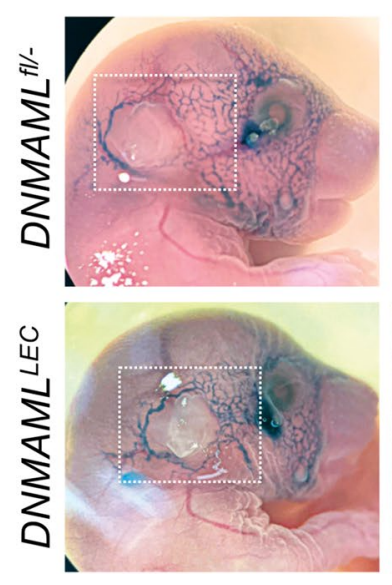

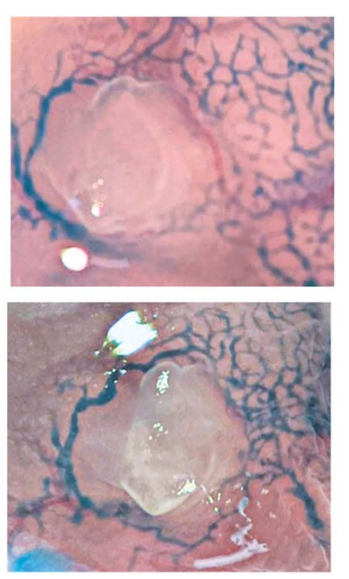

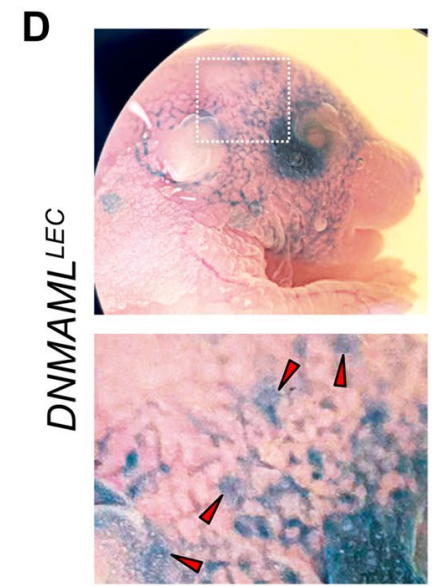

in LEC migration toward the midline. In HdLECs, ectopic Notch 4 activation inhibited LEC migration significantly more than Notch1 activation. This inhibition of LEC migration by Notch4 may occur via non-canonical Notch signaling, as expression of DNMAML, an inhibitor of canonical Notch signaling, did not affect the closure of the lymphangiogenic fronts. Notch4 may suppress LEC migration via its interactions with $\mathrm{Wnt} / \beta$-catenin signaling. Non-canonical Notch4 signaling has been shown to antagonize $\mathrm{Wnt} / \beta$ catenin signaling in stem and progenitor cells [29, 51]. In LECs, loss of $\beta$-catenin signaling reduced LEC migration toward the midline and increased dermal lymphatic vessel caliber [29,51], phenotypes opposite to that observed in Notch $^{-/-}$embryos, suggesting that Notch4 via a non-canonical signaling suppresses LEC migration.

Western analysis of embryo lysates and immunostaining of tissue sections using an antibody against the cytoplasmic domain of NOTCH4 demonstrated a loss of NOTCH4 expression in the Notch4 nulls. It has been suggested that this Notch4 null line expresses a truncated extracellular
NOTCH4 peptide that suppresses Notch1 signaling by functioning as a ligand trap [52]. However, a loss of canonical Notch signaling was not observed in the lymphatics of Notch4 mutant mice, which would be predicted if Notch1 signaling was inhibited in the model. Moreover, the Notch4 mutant dermal lymphatic phenotype is distinct from that observed in mice with Notchl deleted in the LECs [14], as well as the DNMAML ${ }^{L E C}$ mice. The dermal lymphatic phenotype however may be due to loss of Notch4 in non-LECs, such as macrophages, and a conditional Notch4 allele needs to be developed to better understand the cell type specific requirement for NOTCH4 in lymphatic development.

Together with published data, our studies suggest that Notch1 and Notch4 function distinctly in embryonic dermal lymphangiogenesis via a RBPjk-dependent and -independent pathways. We propose that Dll4/Notch1 signaling via a canonical pathway suppresses LEC proliferation, while Notch4 signaling suppresses LEC migration and branching, possibly via a RBPjк-dependent mechanism. Further studies into the mechanistic interaction between Notch1 and Notch4 
Fig. 9 Canonical Notch signaling in LECs is unaltered in the Notch4 mutant dermal lymphatics. Notch $^{+/-} ;$ProxTOM $^{+/} ; \mathrm{NVR}^{+/-}$ males were bred with Notch $^{+/-} ;$ProxTom $^{+/} ; \mathrm{NVR}^{+/-}$ females, and E14.5 ProxTom; NVR and Notch4 ${ }^{-/} ;$ProxTom;NVR dermal wholemounts stained for LYVE1. a Representative images of blunted-ended and spiky-ended sprouts at the lymphangiogenic front of wild-type (WT) and Notch4 ${ }^{-/-}$dermis. b Representative images of branch-points with Notch signaling in WT and $\mathrm{Notch}^{-/-}$in the maturing lymphatic vascular plexus. Scale bars, $50 \mu \mathrm{m}$
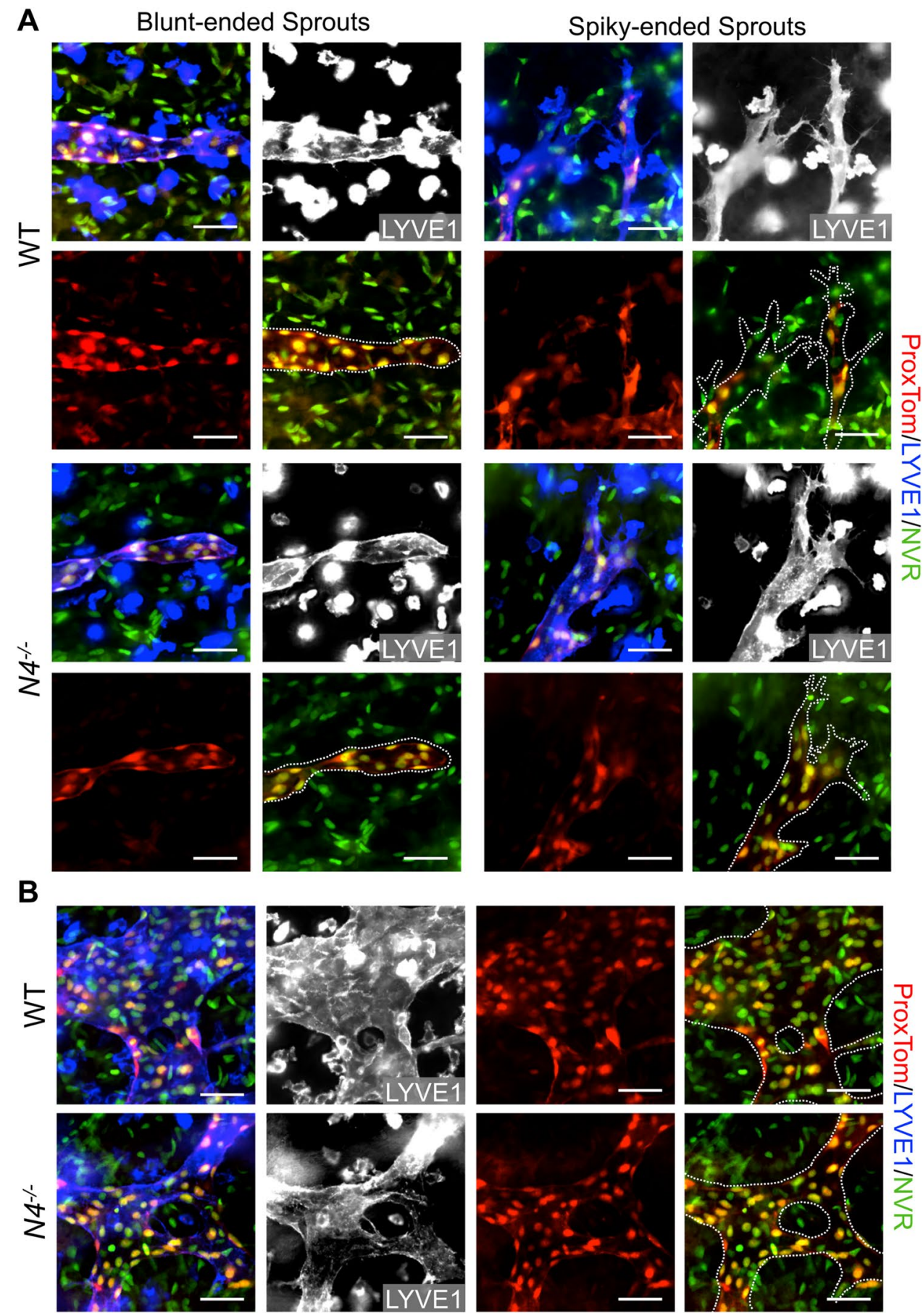

in LECs and lymphatic development and homeostasis are necessary, as a number of therapeutics that are pan-Notch inhibitors or target specific receptors or ligands are currently in clinical trials or the research pipeline for use in the clinic.

Supplementary Information The online version contains supplementary material available at https://doi.org/10.1007/s10456-021-09822-5.
Acknowledgements The authors thank Valeriya Borisenko and Marina Vorontchikhina for technical assistance, June Wu for critical reading of the manuscript, and Warren Pear $\left(D N M A M L^{f l f l}\right)$, Tom Gridley $\left(\right.$ Notch $\left.^{-/-}\right)$, Guillermo Oliver (ProxlCreER ${ }^{T 2}$ ), and Hong Young Kwon (Proxl-tdTomato) for providing mice.

Author contributions AM and MKU share first authorship. AM, MKU, YM, JKK, CJS contributed to the study conception and design. Material preparation, data collection, and analysis were performed by AM, 
MKU, GSD, BS, JMJ, AM, SWY, JDM, CK, MG, GR, CJS. The first draft of the manuscript was written by AM, MKU, and CJS and revised by CJS.

Funding This study was funded by the NIH/NCI (R01CA136673; CJS, JKK), NIH/NIDDK (R01 R01DK107633; CJS), NIH/ NHLBI (RO1HL112626; JKK), the DOD pre-doctoral fellowship (W81XWH-10-1-0304; MKU), and the Lipedema Foundation (CJS). These studies used the resources of the Herbert Irving Comprehensive Cancer Center Flow Cytometry Shared Resources funded in part through Center Grant P30CA013696.

\section{Declarations}

Conflict of interest Jan Kitajewski has received research funding from Eisai Pharmaceuticals (CU12-3625 and UICID\#084028 Eisai Ltd. Research Collaborative Agreements). All other authors declare that they have no conflict of interests.

Ethical approval Isolation of HUVEC and HdLEC from anonymous discarded specimens and received IRB exempt status by Columbia University IRB (AAAA7338). All procedures performed in studies involving human participants were in accordance with the ethical standards of the institutional and/or national research committee and with the 1964 Helsinki declaration and its later amendments or comparable ethical standards. Mouse studies were approved by Columbia University IACUC (AC-AAAE2653, AC-AAAD0577, AC-AAAP9603, ACAAAP0452, AC-AABB9551). All procedures performed in studies involving animals were in accordance with the ethical standards of the institution or practice at which the studies were conducted.

Open Access This article is licensed under a Creative Commons Attribution 4.0 International License, which permits use, sharing, adaptation, distribution and reproduction in any medium or format, as long as you give appropriate credit to the original author(s) and the source, provide a link to the Creative Commons licence, and indicate if changes were made. The images or other third party material in this article are included in the article's Creative Commons licence, unless indicated otherwise in a credit line to the material. If material is not included in the article's Creative Commons licence and your intended use is not permitted by statutory regulation or exceeds the permitted use, you will need to obtain permission directly from the copyright holder. To view a copy of this licence, visit http://creativecommons.org/licenses/by/4.0/.

\section{References}

1. James JM, Nalbandian A, Mukouyama YS (2013) TGFbeta signaling is required for sprouting lymphangiogenesis during lymphatic network development in the skin. Development 140(18):39033914. doi:https://doi.org/10.1242/dev.095026

2. D'Souza B, Meloty-Kapella L, Weinmaster G (2010) Canonical and non-canonical Notch ligands. Curr Top Dev Biol 92:73-129. doi:https://doi.org/10.1016/S0070-2153(10)92003-6

3. Baeten JT, Lilly B (2017) Notch signaling in vascular smooth muscle cells. Adv Pharmacol 78:351-382. https://doi.org/10. 1016/bs.apha.2016.07.002

4. Li JL, Harris AL (2009) Crosstalk of VEGF and Notch pathways in tumour angiogenesis: therapeutic implications. Front Biosci 14:3094-3110

5. Shawber CJ, Kitajewski J (2004) Notch function in the vasculature: insights from zebrafish, mouse and man. BioEssays: news and reviews in molecular. Cell Dev Biol 26(3):225-234. https:// doi.org/10.1002/bies.20004

6. Hellstrom M, Phng LK, Hofmann JJ, Wallgard E, Coultas L, Lindblom P, Alva J, Nilsson AK, Karlsson L, Gaiano N, Yoon K, Rossant J, Iruela-Arispe ML, Kalen M, Gerhardt H, Betsholtz C (2007) Dl14 signalling through Notch1 regulates formation of tip cells during angiogenesis. Nature 445(7129):776-780. doi:https:// doi.org/10.1038/nature05571

7. Lobov IB, Renard RA, Papadopoulos N, Gale NW, Thurston G, Yancopoulos GD, Wiegand SJ (2007) Delta-like ligand 4 (Dll4) is induced by VEGF as a negative regulator of angiogenic sprouting. Proc Natl Acad Sci USA 104(9):3219-3224. doi:https://doi.org/ 10.1073/pnas.0611206104

8. Suchting S, Freitas C, le Noble F, Benedito R, Breant C, Duarte A, Eichmann A (2007) The Notch ligand Delta-like 4 negatively regulates endothelial tip cell formation and vessel branching. Proc Natl Acad Sci USA 104(9):3225-3230. doi:https://doi.org/ 10.1073/pnas.0611177104

9. Tammela T, Zarkada G, Wallgard E, Murtomaki A, Suchting S, Wirzenius M, Waltari M, Hellstrom M, Schomber T, Peltonen R, Freitas C, Duarte A, Isoniemi H, Laakkonen P, Christofori G, Yla-Herttuala S, Shibuya M, Pytowski B, Eichmann A, Betsholtz C, Alitalo K (2008) Blocking VEGFR-3 suppresses angiogenic sprouting and vascular network formation. Nature 454(7204):656660. doi:https://doi.org/10.1038/nature07083

10. Bernier-Latmani J, Cisarovsky C, Demir CS, Bruand M, Jaquet M, Davanture S, Ragusa S, Siegert S, Dormond O, Benedito R, Radtke F, Luther SA, Petrova TV (2015) DLL4 promotes continuous adult intestinal lacteal regeneration and dietary fat transport. J Clin Investig 125(12):4572-4586. doi:https://doi. org/10.1172/JCI82045

11. Zheng W, Tammela T, Yamamoto M, Anisimov A, Holopainen T, Kaijalainen S, Karpanen T, Lehti K, Yla-Herttuala S, Alitalo K (2011) Notch restricts lymphatic vessel sprouting induced by vascular endothelial growth factor. Blood 118(4):1154-1162. doi:https://doi.org/10.1182/blood-2010-11-317800

12. Shawber CJ, Funahashi Y, Francisco E, Vorontchikhina M, Kitamura Y, Stowell SA, Borisenko V, Feirt N, Podgrabinska S, Shiraishi K, Chawengsaksophak K, Rossant J, Accili D, Skobe M, Kitajewski J (2007) Notch alters VEGF responsiveness in human and murine endothelial cells by direct regulation of VEGFR-3 expression. J Clin Investig 117(11):3369-3382. doi:https://doi.org/10.1172/JCI24311

13. Niessen K, Zhang G, Ridgway JB, Chen H, Kolumam G, Siebel CW, Yan M (2011) The Notch1-Dll4 signaling pathway regulates mouse postnatal lymphatic development. Blood 118(7):1989-1997. doi:https://doi.org/10.1182/ blood-2010-11-319129

14. Fatima A, Culver A, Culver F, Liu T, Dietz WH, Thomson BR, Hadjantonakis AK, Quaggin SE, Kume T (2014) Murine Notch1 is required for lymphatic vascular morphogenesis during development. Dev Dyn 243(7):957-964. https://doi.org/10.1002/dvdy.24129

15. Geng X, Yanagida K, Akwii RG, Choi D, Chen L, Ho Y, Cha B, Mahamud MR, Berman de Ruiz K, Ichise H, Chen H, Wythe JD, Mikelis CM, Hla T, Srinivasan RS (2020) S1PR1 regulates the quiescence of lymphatic vessels by inhibiting laminar shear stress-dependent VEGF-C signaling. JCI Insight 5(14):e137652

16. Funahashi Y, Shawber CJ, Sharma A, Kanamaru E, Choi YK, Kitajewski J (2011) Notch modulates VEGF action in endothelial cells by inducing matrix metalloprotease activity. Vasc Cell 3(1):2. https://doi.org/10.1186/2045-824X-3-2

17. Funahashi Y, Shawber CJ, Vorontchikhina M, Sharma A, Outtz HH, Kitajewski J (2010) Notch regulates the angiogenic response via induction of VEGFR-1. J Angiogenesis Res 2(1):3. https://doi. org/10.1186/2040-2384-2-3 
18. Murtomaki A, Uh MK, Choi YK, Kitajewski C, Borisenko V, Kitajewski J, Shawber CJ (2013) Notch1 functions as a negative regulator of lymphatic endothelial cell differentiation in the venous endothelium. Development 140(11):2365-2376. doi:https://doi.org/10.1242/dev.083865

19. Tung JJ, Hobert O, Berryman M, Kitajewski J (2009) Chloride intracellular channel 4 is involved in endothelial proliferation and morphogenesis in vitro. Angiogenesis 12(3):209-220. doi:https:// doi.org/10.1007/s10456-009-9139-3

20. Uyttendaele H, Marazzi G, Wu G, Yan Q, Sassoon D, Kitajewski J (1996) Notch4/Int-3, a mammary proto-oncogene, is an endothelial cell-specific mammalian Notch gene. Development 122(7):2251-2259

21. Geback T, Schulz MM, Koumoutsakos P, Detmar M (2009) TScratch: a novel and simple software tool for automated analysis of monolayer wound healing assays. Biotechniques 46(4):265274. doi:https://doi.org/10.2144/000113083

22. Schmittgen TD, Livak KJ (2008) Analyzing real-time PCR data by the comparative C(T) method. Nat Protoc 3(6):1101-1108. https://doi.org/10.1038/nprot.2008.73

23. Shawber CJ, Das I, Francisco E, Kitajewski J (2003) Notch signaling in primary endothelial cells. Ann N Y Acad Sci 995:162-170. doi:https://doi.org/10.1111/j.1749-6632.2003.tb03219.x

24. Krebs LT, Xue Y, Norton CR, Shutter JR, Maguire M, Sundberg JP, Gallahan D, Closson V, Kitajewski J, Callahan R, Smith GH, Stark KL, Gridley T (2000) Notch signaling is essential for vascular morphogenesis in mice. Genes Dev 14(11):1343-1352

25. Srinivasan RS, Dillard ME, Lagutin OV, Lin FJ, Tsai S, Tsai MJ, Samokhvalov IM, Oliver G (2007) Lineage tracing demonstrates the venous origin of the mammalian lymphatic vasculature. Genes Dev 21(19):2422-2432. doi:https://doi.org/10.1101/gad.1588407

26. Tu L, Fang TC, Artis D, Shestova O, Pross SE, Maillard I, Pear WS (2005) Notch signaling is an important regulator of type 2 immunity. J Exp Med 202(8):1037-1042. https://doi.org/10.1084/jem.20050923

27. Nowotschin S, Xenopoulos P, Schrode N, Hadjantonakis AK (2013) A bright single-cell resolution live imaging reporter of Notch signaling in the mouse. BMC Dev Biol 13:15. doi:https://doi.org/10.1186/ 1471-213X-13-15

28. Hong M, Jung E, Yang S, Jung W, Seong YJ, Park E, Bramos A, Kim KE, Lee S, Daghlian G, Seo JI, Choi I, Choi IS, Koh CJ, Kobielak A, Ying QL, Johnson M, Gardner D, Wong AK, Choi D, Hong YK (2016) Efficient assessment of developmental, surgical and pathological lymphangiogenesis using a lymphatic reporter mouse and its embryonic stem cells. PloS One 11(6):e0157126. https://doi. org/10.1371/journal.pone.0157126

29. Cha B, Geng X, Mahamud MR, Fu J, Mukherjee A, Kim Y, Jho EH, Kim TH, Kahn ML, Xia L, Dixon JB, Chen H, Srinivasan RS (2016) Mechanotransduction activates canonical Wnt/beta-catenin signaling to promote lymphatic vascular patterning and the development of lymphatic and lymphovenous valves. Genes Dev 30(12):1454-1469. doi:https://doi.org/10.1101/gad.282400.116

30. Vorontchikhina MA, Zimmermann RC, Shawber CJ, Tang H, Kitajewski J (2005) Unique patterns of Notch1, Notch4 and Jagged1 expression in ovarian vessels during folliculogenesis and corpus luteum formation. Gene Expr Patterns 5(5):701-709. doi:https:// doi.org/10.1016/j.modgep.2005.02.001

31. Du LL, Liu P (2016) CXCL12/CXCR4 axis regulates neovascularization and lymphangiogenesis in sutured corneas in mice. Mol Med Rep 13(6):4987-4994. doi:https://doi.org/10.3892/mmr.2016.5179

32. Abengozar MA, de Frutos S, Ferreiro S, Soriano J, Perez-Martinez M, Olmeda D, Marenchino M, Canamero M, Ortega S, Megias D, Rodriguez A, Martinez-Torrecuadrada JL (2012) Blocking ephrinB2 with highly specific antibodies inhibits angiogenesis, lymphangiogenesis, and tumor growth. Blood 119(19):4565-4576. doi:https:// doi.org/10.1182/blood-2011-09-380006
33. Farnsworth RH, Karnezis T, Maciburko SJ, Mueller SN, Stacker SA (2019) The interplay between lymphatic vessels and chemokines. Front Immunol 10:518. https://doi.org/10.3389/fimmu.2019.00518

34. Kanady JD, Dellinger MT, Munger SJ, Witte MH, Simon AM (2011) Connexin37 and connexin43 deficiencies in mice disrupt lymphatic valve development and result in lymphatic disorders including lymphedema and chylothorax. Dev Biol 354(2):253-266. https://doi.org/10.1016/j.ydbio.2011.04.004

35. Klein KR, Karpinich NO, Espenschied ST, Willcockson HH, Dunworth WP, Hoopes SL, Kushner EJ, Bautch VL, Caron KM (2014) Decoy receptor CXCR7 modulates adrenomedullin-mediated cardiac and lymphatic vascular development. Dev Cell 30(5):528-540. https://doi.org/10.1016/j.devcel.2014.07.012

36. Lee KM, Danuser R, Stein JV, Graham D, Nibbs RJ, Graham GJ (2014) The chemokine receptors ACKR2 and CCR2 reciprocally regulate lymphatic vessel density. EMBO J 33(21):2564-2580. doi:https://doi.org/10.15252/embj.201488887

37. Uchida Y, James JM, Suto F, Mukouyama YS (2015) Class 3 semaphorins negatively regulate dermal lymphatic network formation. Biol Open 4(9):1194-1205. doi:https://doi.org/10.1242/bio.012302

38. Zhuo W, Jia L, Song N, Lu XA, Ding Y, Wang X, Song X, Fu Y, Luo Y (2012) The CXCL12-CXCR4 chemokine pathway: a novel axis regulates lymphangiogenesis. Clin Cancer Res 18(19):5387-5398. doi:https://doi.org/10.1158/1078-0432.CCR-12-0708

39. Karpinich NO, Caron KM (2014) Apelin signaling: new G proteincoupled receptor pathway in lymphatic vascular development. Arterioscler Thromb Vasc Biol 34(2):239-241. doi:https://doi.org/10. 1161/ATVBAHA.113.302905

40. Lohela M, Saaristo A, Veikkola T, Alitalo K (2003) Lymphangiogenic growth factors, receptors and therapies. Thromb Haemost 90(2):167-184. doi:https://doi.org/10.1160/TH03-04-0200

41. Benedito R, Rocha SF, Woeste M, Zamykal M, Radtke F, Casanovas O, Duarte A, Pytowski B, Adams RH (2012) Notch-dependent VEGFR3 upregulation allows angiogenesis without VEGFVEGFR2 signalling. Nature 484(7392):110-114. doi:https://doi. org/10.1038/nature10908

42. Costa MJ, Wu X, Cuervo H, Srinivasan R, Bechis SK, Cheang E, Marjanovic O, Gridley T, Cvetic CA, Wang RA (2013) Notch4 is required for tumor onset and perfusion. Vasc Cell 5(1):7. https://doi. org/10.1186/2045-824X-5-7

43. Zhang Y, Ulvmar MH, Stanczuk L, Martinez-Corral I, Frye M, Alitalo K, Makinen T (2018) Heterogeneity in VEGFR3 levels drives lymphatic vessel hyperplasia through cell-autonomous and non-cellautonomous mechanisms. Nat Commun 9(1):1296. doi:https://doi. org/10.1038/s41467-018-03692-0

44. Martinez-Corral I, Zhang Y, Petkova M, Ortsater H, Sjoberg S, Castillo SD, Brouillard P, Libbrecht L, Saur D, Graupera M, Alitalo K, Boon L, Vikkula M, Makinen T (2020) Blockade of VEGF-C signaling inhibits lymphatic malformations driven by oncogenic PIK3CA mutation. Nat Commun 11(1):2869. doi:https://doi.org/ 10.1038/s41467-020-16496-y

45. MacKenzie F, Duriez P, Wong F, Noseda M, Karsan A (2004) Notch4 inhibits endothelial apoptosis via RBP-Jkappa-dependent and -independent pathways. J Biol Chem 279(12):11657-11663. doi:https://doi.org/10.1074/jbc.M312102200

46. Raafat A, Bargo S, McCurdy D, Callahan R (2017) The ANK repeats of Notch-4/Int3 activate NF-kappaB canonical pathway in the absence of Rbpj and causes mammary tumorigenesis. Scientific reports 7(1):13690. doi:https://doi.org/10.1038/s41598-017-13989-7

47. Raafat A, Lawson S, Bargo S, Klauzinska M, Strizzi L, Goldhar AS, Buono K, Salomon D, Vonderhaar BK, Callahan R (2009) Rbpj conditional knockout reveals distinct functions of Notch4/ Int3 in mammary gland development and tumorigenesis. Oncogene 28(2):219-230. doi:https://doi.org/10.1038/onc.2008.379

48. Liu X, Luo Q, Zheng Y, Liu X, Hu Y, Liu W, Luo M, Zhao Y, Zou L (2016) NOTCH4 signaling controls EFNB2-induced 
endothelial progenitor cell dysfunction in preeclampsia. Reproduction 152(1):47-55. doi:https://doi.org/10.1530/REP-16-0132

49. Pedrosa AR, Trindade A, Fernandes AC, Carvalho C, Gigante J, Tavares AT, Dieguez-Hurtado R, Yagita H, Adams RH, Duarte A (2015) Endothelial Jagged1 antagonizes Dl14 regulation of endothelial branching and promotes vascular maturation downstream of Dll4/Notch1. Arterioscler Thromb Vasc Biol 35(5):1134-1146. https://doi.org/10.1161/ATVBAHA.114.304741

50. Murtomaki A, Uh MK, Kitajewski C, Zhao J, Nagasaki T, Shawber CJ, Kitajewski J (2014) Notch signaling functions in lymphatic valve formation. Development 141(12):2446-2451. doi:https://doi.org/10. 1242/dev. 101188
51. Andersen P, Uosaki H, Shenje LT, Kwon C (2012) Non-canonical Notch signaling: emerging role and mechanism. Trends Cell Biol 22(5):257-265. doi:https://doi.org/10.1016/j.tcb.2012.02.003

52. James AC, Szot JO, Iyer K, Major JA, Pursglove SE, Chapman G, Dunwoodie SL (2014) Notch4 reveals a novel mechanism regulating Notch signal transduction. Biochim Biophys Acta 1843(7):12721284. doi:https://doi.org/10.1016/j.bbamcr.2014.03.015

Publisher's Note Springer Nature remains neutral with regard to jurisdictional claims in published maps and institutional affiliations. 